\title{
Determination of $\alpha_{S}$ using the Next-to-Leading-Log Approximation of QCD
}

DELPHI Collaboration

\begin{abstract}
A new measurement of $\alpha_{S}$ is obtained from the distributions in thrust, heavy jet mass, energy-energy correlation and two recently introduced jet broadening variables following a method proposed by Catani, Trentadue, Turnock and Webber. This method includes the full calculation of $\mathcal{O}\left(\alpha_{S}^{2}\right)$ terms and leading and next-to-leading logarithms resummed to all orders of $\alpha_{S}$. The analysis is based on data taken with the DELPHI detector at LEP during 1991.

It is found that the inclusion of the resummed leading and next-to-leading logarithms reduces the scale dependence of $\alpha_{S}$ and allows an extension of the fit range towards the infrared limit of the kinematical range. The combined value for $\alpha_{S}$ obtained at the scale $\mu^{2}=M_{\mathrm{Z}}^{2}$ is :
\end{abstract}

$$
\alpha_{S}\left(M_{\mathrm{Z}}^{2}\right)=0.123 \pm 0.006
$$




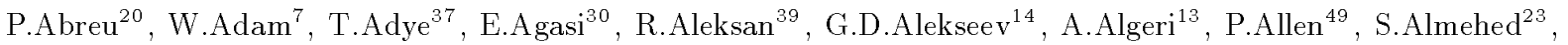
S.J.Alvsvaag ${ }^{4}$ U.Amaldi ${ }^{7}, \quad$ A.Andreazza ${ }^{27}, \quad$ P.Antilogus ${ }^{24}$, W-D.Apel ${ }^{15}$, R.J.Apsimon ${ }^{37}, \quad$ Y.Arnoud $^{39}$, B.Asman ${ }^{45}$, J-E.Augustin ${ }^{18}$, A.Augustinus ${ }^{30}$, P.Baillon ${ }^{7}$, P.Bambade $^{18}$, F.Barao $^{20}$, R.Barate ${ }^{12}$, G.Barbiellini ${ }^{47}$, D.Y.Bardin ${ }^{14}$, G.J.Barker ${ }^{34}$, A.Baroncelli ${ }^{41}$, O.Barring ${ }^{7}$, J.A.Barrio ${ }^{25}$, W.Bartl ${ }^{50}$, M.J.Bates ${ }^{37}$, M.Battaglia $^{13}$, M.Baubillier ${ }^{22}$, K-H.Becks ${ }^{52}$, C.J.Beeston ${ }^{34}, \quad$ M.Begalli ${ }^{36}$, P.Beilliere ${ }^{6}$, Yu.Belokopytov ${ }^{43}$, P.Beltran ${ }^{9}$, D.Benedic $^{8}$, A.C.Benvenutit ${ }^{5}$, M.Berggren ${ }^{18}$, D.Bertrand ${ }^{2}$, F.Bianchi' ${ }^{46}$, M.S.Bilenky ${ }^{14}$, P.Billoir ${ }^{22}$, J.Bjarne ${ }^{23}$,

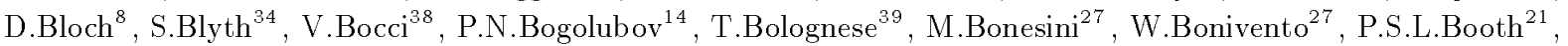
G.Borisov $^{43}$, H.Borner ${ }^{7}$, C.Bosio ${ }^{41}$, B.Bostjancic ${ }^{44}$, S.Bosworth $^{34}$, O.Botner ${ }^{48}$, B.Bouquet ${ }^{18}$, C.Bourdarios ${ }^{18}$, T.J.V.Bowcock ${ }^{21}$, M.Bozzo ${ }^{11}$, S.Braibant ${ }^{2}$, P.Branchini ${ }^{41}$, K.D.Brand ${ }^{35}$, R.A.Brenner ${ }^{7}$, H.Briand ${ }^{22}$, C.Bricman $^{2}$, R.C.A.Brown ${ }^{7}$, N.Brummer ${ }^{30}$, J-M.Brunet ${ }^{6}$, L.Bugge ${ }^{32}$, T.Buran ${ }^{32}$, H.Burmeister ${ }^{7}$, J.A.M.A.Buytaert ${ }^{7}$, M.Caccia ${ }^{7}$, M.Calvi ${ }^{27}$, A.J.Camacho Rozas ${ }^{42}$, R.Campion ${ }^{21}$, T.Camporesi ${ }^{7}$, V.Canale ${ }^{38}$, F.Cao $^{2}$, F.Carena ${ }^{7}$, L.Carroll ${ }^{21}$, C.Caso ${ }^{11}$, M.V.Castillo Gimenez ${ }^{49}$, A.Cattai ${ }^{7}$, F.R.Cavallo ${ }^{5}$, L.Cerrito ${ }^{38}$, V.Chabaud ${ }^{7}$, A.Chan $^{1}$, M.Chapkin ${ }^{43}$, Ph.Charpentier ${ }^{7}$, L.Chaussard ${ }^{18}$, J.Chauveau ${ }^{22}$, P.Checchia ${ }^{35}$, G.A.Chelkov ${ }^{14}$, L.Chevalier ${ }^{39}$, P.Chliapnikov ${ }^{43}$, V.Chorowicz ${ }^{22}$, J.T.M.Chrin ${ }^{49}$, M.P.Clara ${ }^{46}$, P.Collins ${ }^{34}$, J.L.Contreras ${ }^{25}$, R.Contri ${ }^{11}$, E.Cortina ${ }^{49}$, G.Cosme $^{18}$, F.Couchot ${ }^{18}$, H.B.Crawley ${ }^{1}$, D.Crennell ${ }^{37}$, G.Crosetti $^{11}$, M.Crozon ${ }^{6}$, J.Cuevas Maestro ${ }^{33}$, S.Czellar ${ }^{13}$, E.Dahl-Jensen ${ }^{28}$, B.Dalmagne ${ }^{18}$, M.Dam ${ }^{32}$, G.Damgaard ${ }^{28}$, G.Darbo $^{11}$, E.Daubie ${ }^{2}$, A.Daum ${ }^{15}$, P.D.Dauncey ${ }^{34}$, M.Davenport ${ }^{7}$, P.David ${ }^{22}$, J.Davies ${ }^{21}$, W.Da Silva ${ }^{22}$, C.Defoix $^{6}$, P.Delpierre ${ }^{6}$, N.Demaria ${ }^{46}$, A.De Angelis ${ }^{47}$, H.De Boeck ${ }^{2}$, W.De Boer ${ }^{15}$, C.De Clercq ${ }^{2}$, M.D.M.De Fez Laso ${ }^{49}$, N.De Groot ${ }^{30}$, C.De La Vaissiere ${ }^{22}$, B.De Lotto ${ }^{47}, \quad$ A.De Min ${ }^{27}, \quad$ H.Dijkstra ${ }^{7}$, L.Di Ciaccio ${ }^{38}$, F.Djama ${ }^{8}$, J.Dolbeau ${ }^{6}$, M.Donszelmann $^{7}$, K.Doroba ${ }^{51}$, M.Dracos ${ }^{7}$, J.Drees ${ }^{52}$, M.Dris ${ }^{31}$, Y.Dufour $^{7}$, F.Dupont ${ }^{12}$, L-O.Eek ${ }^{48}$, P.A.-M.Eerola ${ }^{7}$, R.Ehret ${ }^{15}$, T.Ekelof ${ }^{48}$, G.Ekspong ${ }^{45}$, A.Elliot Peisert ${ }^{35}$, J-P.Engel $^{8}$, N.Ershaidat ${ }^{22}$, D.Fassouliotis ${ }^{31}$, M.Feindt ${ }^{7}$, M.Fernandez Alonso ${ }^{42}$, A.Ferrer ${ }^{49}$, T.A.Filippas ${ }^{31}$, A.Firestone $^{1}$, H.Foeth ${ }^{7}$, E.Fokitis ${ }^{31}$, F.Fontanelli ${ }^{11}$, K.A.J.Forbes ${ }^{21}$, J-L.Fousset ${ }^{26}$, S.Francon ${ }^{24}$, B.Franek ${ }^{37}$, P.Frenkiel $^{6}$, D.C.Fries ${ }^{15}$, A.G.Frodesen ${ }^{4}$, R.Fruhwirth ${ }^{50}$, F.Fulda-Quenzer ${ }^{18}$, K.Furnival ${ }^{21}$, H.Furstenau ${ }^{15}$, J.Fuster ${ }^{7}$, D.Gamba ${ }^{46}$, C.Garcia ${ }^{49}$, J.Garcia ${ }^{42}$, C.Gaspar ${ }^{7}$, U.Gasparini ${ }^{35}$, Ph.Gavillet ${ }^{7}$, E.N.Gazis ${ }^{31}$, J-P.Gerber $^{8}$, P.Giacomelli ${ }^{7}$, R.Gokieli ${ }^{51}$, B.Golob ${ }^{44}$, V.M.Golovatyuk ${ }^{14}$, J.J.Gomez Y Cadenas ${ }^{7}$, A.Goobar ${ }^{45}$, G.Gopal $^{37}$, M.Gorski ${ }^{51}$, V.Gracco ${ }^{11}$, A.Grant ${ }^{7}$, F.Grard ${ }^{2}$, E.Graziani ${ }^{41}$, G.Grosdidier ${ }^{18}$, E.Gross ${ }^{7}$, P.Grosse-Wiesmann ${ }^{7}$, B.Grossetete ${ }^{22}$, J.Guy ${ }^{37}$, U.Haedinger ${ }^{15}$, F.Hahn ${ }^{52}$, M.Hahn ${ }^{15}$, S.Haider ${ }^{30}$, Z.Hajduk ${ }^{16}$, A.Hakansson $^{23}$, A.Hallgren ${ }^{48}$, K.Hamacher ${ }^{52}$, G.Hamel De Monchenault ${ }^{39}$, W.Hao ${ }^{30}$, F.J.Harris ${ }^{34}$, V.Hedberg ${ }^{23}$, T.Henkes ${ }^{7}$, J.J.Hernandez ${ }^{49}$, P.Herquet ${ }^{2}$, H.Herr ${ }^{7}$, T.L.Hessing ${ }^{21}$, I.Hietanen ${ }^{13}$, C.O.Higgins ${ }^{21}$, E.Higon ${ }^{49}$, H.J.Hilke ${ }^{7}$, S.D.Hodgson ${ }^{34}$, T.Hofmokl ${ }^{51}$, R.Holmes ${ }^{1}, \quad$ S-O.Holmgren ${ }^{45}$, D.Holthuizen ${ }^{30}$, P.F.Honore ${ }^{6}$, J.E.Hooper ${ }^{28}$, M.Houlden ${ }^{21}$, J.Hrubec ${ }^{50}$, K.Huet ${ }^{2}$, P.O.Hulth ${ }^{45}$, K.Hultqvist ${ }^{45}$, P.Ioannou ${ }^{3}$, P-S.Iversen ${ }^{4}$, J.N.Jackson ${ }^{21}$, P.Jalocha ${ }^{16}, \quad$ G.Jarlskog $^{23}, \quad$ P.Jarry ${ }^{39}$, B.Jean-Marie ${ }^{18}$, E.K.Johansson ${ }^{45}$, D.Johnson ${ }^{21}$, M.Jonker ${ }^{7}$, L.Jonsson ${ }^{23}$, P.Juillot ${ }^{8}$, G.Kalkanis ${ }^{3}$, G.Kalmus ${ }^{37}$, F.Kapusta ${ }^{22}$, M.Karlsson ${ }^{7}$, E.Karvelas $^{9}$, S.Katsanevas ${ }^{3}$, E.C.Katsoufis ${ }^{31}$, R.Keranen ${ }^{7}$, J.Kesteman ${ }^{2}$, B.A.Khomenko ${ }^{14}$, N.N.Khovanski ${ }^{14}$, B.King ${ }^{21}$, N.J.Kjaer ${ }^{7}$, H.Klein ${ }^{7}$, A.Klovning ${ }^{4}$, P.Kluit ${ }^{30}$, A.Koch-Mehrin ${ }^{52}$, J.H.Koehne ${ }^{15}$, B.Koene ${ }^{30}$, P.Kokkinias $^{9}$, M.Koratzinos ${ }^{32}$, K.Korcyl ${ }^{16}$, A.V.Korytov ${ }^{14}$, V.Kostioukhine ${ }^{43}$, C.Kourkoumelis ${ }^{3}$, O.Kouznetsov ${ }^{14}$, P.H.Kramer ${ }^{52}$, J.Krolikowski ${ }^{51}$, I.Kronkvist ${ }^{23}$, U.Kruener-Marquis ${ }^{52}$, W.Kucewicz ${ }^{16}$, K.Kulka ${ }^{48}$, K.Kurvinen ${ }^{13}$, C.Lacasta ${ }^{49}$, C.Lambropoulos ${ }^{9}$, J.W.Lamsa ${ }^{1}$, L.Lanceri ${ }^{47}$, V.Lapin ${ }^{43}$, J-P.Laugier ${ }^{39}$, R.Lauhakangas $^{13}$, G.Leder ${ }^{50}$, F.Ledroit ${ }^{12}$, R.Leitner ${ }^{29}$, Y.Lemoigne ${ }^{39}$, J.Lemonne ${ }^{2}$, G.Lenzen ${ }^{\text {52 }}$, V.Lepeltier ${ }^{18}$,

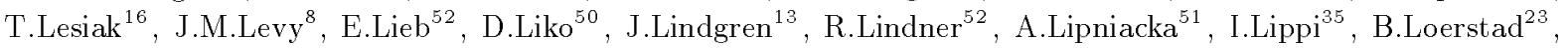
M.Lokajicek ${ }^{10}$, J.G.Loken ${ }^{34}$, A.Lopez-Fernandez ${ }^{7}$, M.A.Lopez Aguera ${ }^{42}$, M.Los ${ }^{30}$, D.Loukas ${ }^{9}$, J.J.Lozano ${ }^{49}$,

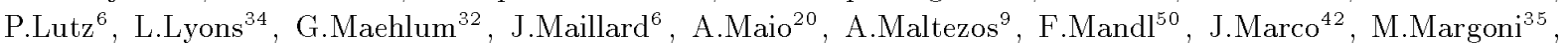
J-C.Marin ${ }^{7}$, A.Markou ${ }^{9}$, T.Maron ${ }^{52}$, S.Marti ${ }^{49}$, L.Mathis ${ }^{1}$, F.Matorras ${ }^{42}$, C.Matteuzzi ${ }^{27}$, G.Matthiae $^{38}$, M.Mazzucato $^{35}$, M.Mc Cubbin ${ }^{21}$, R.Mc Kay ${ }^{1}$, R.Mc Nulty ${ }^{21}$, G.Meola ${ }^{11}$, C.Meroni ${ }^{27}$, W.T.Meyer ${ }^{1}$, M.Michelotto $^{35}$, I.Mikulec ${ }^{50}$, L.Mirabito ${ }^{24}$, W.A.Mitaroff ${ }^{50}$, G.V.Mitselmakher ${ }^{14}$, U.Mjoernmark ${ }^{23}$, T.Moa $^{45}$, R.Moeller $^{28}$, K.Moenig ${ }^{7}$, M.R.Monge ${ }^{11}$, P.Morettini ${ }^{11}$, H.Mueller ${ }^{15}$, W.J.Murray ${ }^{37}$, G.Myatt ${ }^{34}$, F.L.Navarria $^{5}$, P.Negri $^{27}$, R.Nicolaidou ${ }^{3}$, B.S.Nielsen ${ }^{28}$, B.Nijjhar ${ }^{21}$, V.Nikolaenko ${ }^{43}$, P.E.S.Nilsen ${ }^{4}$, P.Niss ${ }^{45}$, A.Nomerotski ${ }^{35}$, V.Obraztsov $^{43}$, A.G.Olshevski ${ }^{14}$, R.Orava ${ }^{13}$, A.Ostankov ${ }^{43}$, K.Osterberg ${ }^{13}$, A.Ouraou ${ }^{39}$, M.Paganoni ${ }^{27}$, R.Pain ${ }^{22}$, H.Palka ${ }^{16}$, Th.D.Papadopoulou ${ }^{31}$, L.Pape ${ }^{7}$, F.Parodi ${ }^{11}$, A.Passeri ${ }^{41}$, M.Pegoraro ${ }^{35}$, J.Pennanen ${ }^{13}$, L.Peralta $^{20}$, V.Perevozchikov ${ }^{43}$, H.Pernegger ${ }^{50}$, M.Pernicka ${ }^{50}$, A.Perrotta ${ }^{5}$, C.Petridou ${ }^{47}$, A.Petrolini ${ }^{11}$, F.Pierre $^{39}$, M.Pimenta ${ }^{20}$, O.Pingot ${ }^{2}$, S.Plaszczynski ${ }^{18}$, O.Podobrin ${ }^{15}$, M.E.Pol ${ }^{7}$, G.Polok ${ }^{16}$, P.Poropat ${ }^{47}$, P.Privitera $^{15}$, A.Pullia ${ }^{27}$, D.Radojicic ${ }^{34}$, S.Ragazzi ${ }^{27}$, H.Rahmani ${ }^{31}$, P.N.Ratoff ${ }^{19}$, A.L.Read ${ }^{32}$, P.Rebecci ${ }^{7}$, N.G.Redaelli ${ }^{27}$, M.Regler ${ }^{50}$, D.Reid ${ }^{7}$, P.B.Renton ${ }^{34}$, L.K.Resvanis ${ }^{3}$, F.Richard ${ }^{18}$, M.Richardson ${ }^{21}$, J.Ridky ${ }^{10}$, G.Rinaudo $^{46}$, I.Roditi ${ }^{17}$, A.Romero ${ }^{46}$, I.Roncagliolo ${ }^{11}$, P.Ronchese ${ }^{35}$, C.Ronnqvist ${ }^{13}$, E.I.Rosenberg ${ }^{1}$, S.Rossi $^{7}$, E.Rosso $^{7}$, P.Roudeau ${ }^{18}$, T.Rovelli ${ }^{5}$, W.Ruckstuhl ${ }^{30}$, V.Ruhlmann-Kleider ${ }^{39}$, A.Ruiz ${ }^{42}$, H.Saarikko ${ }^{13}$, Y.Sacquin ${ }^{39}$, G.Sajot ${ }^{12}$, J.Salt ${ }^{49}$, J.Sanchez ${ }^{25}$, M.Sannino ${ }^{11,40}$, S.Schael ${ }^{7}$, H.Schneider ${ }^{15}$, B.Schulze $^{38}$, M.A.E.Schyns ${ }^{52}$, G.Sciolla ${ }^{46}$,F.Scuri ${ }^{47}$, A.M.Segar ${ }^{34}$, A.Seitz ${ }^{15}$, R.Sekulin ${ }^{37}$, M.Sessa $^{47}$, G.Sette $^{11}$, R.Seufert $^{15}$, 
R.C.Shellard ${ }^{36}$, I.Siccama ${ }^{30}$, P.Siegrist ${ }^{39}$, S.Simonetti ${ }^{11}$, F.Simonetto ${ }^{35}$, A.N.Sisakian ${ }^{14}$, G.Skjevling ${ }^{32}$, G.Smadja $^{39,24}$, N.Smirnov ${ }^{43}$, G.R.Smith ${ }^{37}$, R.Sosnowski ${ }^{51}$, D.Souza-Santos ${ }^{36}$, T.S.Spassoff ${ }^{12}$, E.Spiriti ${ }^{41}$, S.Squarcia ${ }^{11}$, H.Staeck ${ }^{52}$, C.Stanescu ${ }^{41}$, S.Stapnes ${ }^{32}$, G.Stavropoulos ${ }^{9}$, F.Stichelbaut ${ }^{2}$, A.Stocchi ${ }^{18}$, J.Strauss ${ }^{50}$, J.Straver $^{7}$, R.Strub ${ }^{8}$, B.Stugu ${ }^{4}$, M.Szczekowski ${ }^{7}$, M.Szeptycka ${ }^{51}$, P.Szymanski ${ }^{51}$, T.Tabarelli ${ }^{27}$, O.Tchikilev ${ }^{43}$, G.E.Theodosiou ${ }^{9}$, A.Tilquin ${ }^{26}$, J.Timmermans ${ }^{30}$, V.G.Timofeev ${ }^{14}$, L.G.Tkatchev ${ }^{14}$, T.Todorov ${ }^{8}$, D.Z.Toet ${ }^{30}$, O.Toker ${ }^{13}$, B.Tome ${ }^{20}$, E.Torassa ${ }^{46}$, L.Tortora ${ }^{41}$, D.Treille ${ }^{7}$, U.Trevisan ${ }^{11}$, W.Trischuk ${ }^{7}$, G.Tristram ${ }^{6}$, C.Troncon ${ }^{27}$, A.Tsirou ${ }^{7}$, E.N.Tsyganov ${ }^{14}$, M-L.Turluer ${ }^{39}$, T.Tuuva ${ }^{13}$, I.A.Tyapkin ${ }^{22}$, M.Tyndel ${ }^{37}$, S.Tzamarias ${ }^{21}$, S.Ueberschaer ${ }^{52}$, O.Ullaland ${ }^{7}$, V.Uvarov $^{43}$, G.Valenti ${ }^{5}$, E.Vallazza ${ }^{46}$, J.A.Valls Ferrer $^{49}$, C.Vander Velde ${ }^{2}$, G.W.Van Apeldoorn ${ }^{30}$, P.Van Dam ${ }^{30}$, M.Van Der Heijden ${ }^{30}$, W.K.Van Doninck ${ }^{2}$, P.Vaz ${ }^{7}$, G.Vegni $^{27}$, L.Ventura ${ }^{35}$, W.Venus ${ }^{37}$, F.Verbeure ${ }^{2}$, M.Verlato ${ }^{35}$, L.S.Vertogradov ${ }^{14}$, D.Vilanova ${ }^{39}$, P.Vincent $^{24}$, L.Vitale $^{13}$, E.Vlasov ${ }^{43}$, A.S.Vodopyanov ${ }^{14}$, M.Vollmer ${ }^{52}$, M.Voutilainen ${ }^{13}$, V.Vrba ${ }^{41}$, H.Wahlen ${ }^{52}$, C.Walck $^{45}$, F.Waldner ${ }^{47}$, M.Wayne ${ }^{1}$, A.Wehr ${ }^{52}$, M.Weierstall ${ }^{52}$, P.Weilhammer ${ }^{7}$, J.Werner ${ }^{52,}$ A.M.Wetherell ${ }^{7}$, J.H.Wickens ${ }^{2}$, G.R.Wilkinson ${ }^{34}$, W.S.C.Williams ${ }^{34}$, M.Winter ${ }^{8}$, M.Witek ${ }^{16}$, G.Wormser ${ }^{18}$, K.Woschnagg ${ }^{48}$, N.Yamdagni $^{45}$, P.Yepes ${ }^{7}$, A.Zaitsev ${ }^{43}$, A.Zalewska ${ }^{16}$, P.Zalewski ${ }^{18}$, D.Zavrtanik ${ }^{44}$, E.Zevgolatakos ${ }^{9}$, G.Zhang $^{52}$, N.I.Zimin ${ }^{14}$, M.Zito ${ }^{39}$, R.Zuberi ${ }^{34}$, R.Zukanovich Funchal ${ }^{6}$, G.Zumerle ${ }^{35}$, J.Zuniga ${ }^{49}$

\footnotetext{
${ }^{1}$ Ames Laboratory and Department of Physics, Iowa State University, Ames IA 50011, USA

${ }^{2}$ Physics Department, Univ. Instelling Antwerpen, Universiteitsplein 1, B-2610 Wilrijk, Belgium and IIHE, ULB-VUB, Pleinlaan 2, B-1050 Brussels, Belgium

and Faculté des Sciences, Univ. de l'Etat Mons, Av. Maistriau 19, B-7000 Mons, Belgium

${ }^{3}$ Physics Laboratory, University of Athens, Solonos Str. 104, GR-10680 Athens, Greece

${ }^{4}$ Department of Physics, University of Bergen, Allégaten 55, N-5007 Bergen, Norway

${ }^{5}$ Dipartimento di Fisica, Università di Bologna and INFN, Via Irnerio 46, I-40126 Bologna, Italy

${ }^{6}$ Collège de France, Lab. de Physique Corpusculaire, IN2P3-CNRS, F-75231 Paris Cedex 05, France

${ }^{7}$ CERN, CH-1211 Geneva 23, Switzerland

${ }^{8}$ Centre de Recherche Nucléaire, IN2P3 - CNRS/ULP - BP20, F-67037 Strasbourg Cedex, France

${ }^{9}$ Institute of Nuclear Physics, N.C.S.R. Demokritos, P.O. Box 60228, GR-15310 Athens, Greece

${ }^{10}$ FZU, Inst. of Physics of the C.A.S. High Energy Physics Division, Na Slovance 2, CS-180 40, Praha 8, Czechoslovakia

${ }^{11}$ Dipartimento di Fisica, Università di Genova and INFN, Via Dodecaneso 33, I-16146 Genova, Italy

12 Institut des Sciences Nucléaires. IN2P3-CNRS, Université de Grenoble 1, F-38026 Grenoble, France

${ }^{13}$ Research Institute for High Energy Physics, SEFT, Siltavuorenpenger 20 C, SF-00170 Helsinki, Finland

${ }^{14}$ Joint Institute for Nuclear Research, Dubna, Head Post Office, P.O. Box 79, 101000 Moscow, Russian Federation

${ }^{15}$ Institut für Experimentelle Kernphysik, Universität Karlsruhe, Postfach 6980, D-7500 Karlsruhe 1, Germany

${ }^{16}$ High Energy Physics Laboratory, Institute of Nuclear Physics, Ul. Kawiory 26 a, PL-30055 Krakow 30, Poland

${ }^{17}$ Centro Brasileiro de Pesquisas Físicas, rua Xavier Sigaud 150, RJ-22290 Rio de Janeiro, Brazil

${ }^{18}$ Université de Paris-Sud, Lab. de l'Accélérateur Linéaire, IN2P3-CNRS, Bat 200, F-91405 Orsay, France

${ }^{19}$ School of Physics and Materials, University of Lancaster, GB - Lancaster LA1 4YB, UK

${ }^{20}$ LIP, IST, FCUL - Av. Elias Garcia, $14-1^{\circ}$, P-1000 Lisboa Codex, Portugal

${ }^{21}$ Department of Physics, University of Liverpool, P.O. Box 147, GB - Liverpool L69 3BX. UK

${ }^{22}$ LPNHE, IN2P3-CNRS, Universités Paris VI et VII, Tour 33 (RdC), 4 place Jussieu, F-75252 Paris Cedex 05, France

${ }^{23}$ Department of Physics, University of Lund, Sölvegatan 14, S-22363 Lund, Sweden

${ }^{24}$ Université Claude Bernard de Lyon, IPNL, IN2P3-CNRS, F-69622 Villeurbanne Cedex, France

${ }^{25}$ Universidad Complutense, Avda. Complutense s/n, E-28040 Madrid, Spain

${ }^{26}$ Univ. d'Aix - Marseille II - CPP, IN2P3-CNRS, F-13288 Marseille Cedex 09, France

${ }^{27}$ Dipartimento di Fisica, Università di Milano and INFN, Via Celoria 16, I-20133 Milan, Italy

${ }^{28}$ Niels Bohr Institute, Blegdamsvej 17, DK-2100 Copenhagen 0, Denmark

${ }^{29}$ NC, Nuclear Centre of MFF, Charles University, Areal MFF, V Holesovickach 2, CS-180 00, Praha 8, Czechoslovakia

${ }^{30}$ NIKHEF-H, Postbus 41882, NL-1009 DB Amsterdam, The Netherlands

31 National Technical University, Physics Department, Zografou Campus, GR-15773 Athens, Greece

32 Physics Department, University of Oslo, Blindern, N-1000 Oslo 3, Norway

${ }^{33}$ Dpto. Fisica, Univ. Oviedo, C/P.Jimenez Casas, S/N-33006 Oviedo, Spain

${ }^{34}$ Nuclear Physics Laboratory, University of Oxford, Keble Road, GB - Oxford OX1 3RH, UK

${ }^{35}$ Dipartimento di Fisica, Università di Padova and INFN, Via Marzolo 8, I-35131 Padua, Italy

${ }^{36}$ Depto. de Fisica, Pontificia Univ. Católica, C.P. 38071 RJ-22453 Rio de Janeiro, Brazil

${ }^{37}$ Rutherford Appleton Laboratory, Chilton, GB - Didcot OX11 OQX, UK

${ }^{38}$ Dipartimento di Fisica, Università di Roma II and INFN, Tor Vergata, I-00173 Rome, Italy

${ }^{39}$ Centre d'Etude de Saclay, DSM/DAPNIA, F-91191 Gif-sur-Yvette Cedex, France

40 Dipartimento di Fisica-Università di Salerno, I-84100 Salerno, Italy

${ }^{41}$ Istituto Superiore di Sanità, Ist. Naz. di Fisica Nucl. (INFN), Viale Regina Elena 299, I-00161 Rome, Italy

${ }^{42}$ C.E.A.F.M., C.S.I.C. - Univ. Cantabria, Avda. los Castros, S/N-39006 Santander, Spain

${ }^{43}$ Inst. for High Energy Physics, Serpukow P.O. Box 35, Protvino, (Moscow Region), Russian Federation

${ }^{44}$ J. Stefan Institute and Department of Physics, University of Ljubljana, Jamova 39, SI-61000 Ljubljana, Slovenia

${ }^{45}$ Institute of Physics, University of Stockholm, Vanadisvägen 9, S-113 46 Stockholm, Sweden

${ }^{46}$ Dipartimento di Fisica Sperimentale, Università di Torino and INFN, Via P. Giuria 1, I-10125 Turin, Italy

${ }^{47}$ Dipartimento di Fisica, Università di Trieste and INFN, Via A. Valerio 2, I-34127 Trieste, Italy and Istituto di Fisica, Università di Udine, I-33100 Udine, Italy

${ }^{48}$ Department of Radiation Sciences, University of Uppsala, P.O. Box 535, S-751 21 Uppsala, Sweden

${ }^{49}$ IFIC, Valencia-CSIC, and D.F.A.M.N., U. de Valencia, Avda. Dr. Moliner 50, E-46100 Burjassot (Valencia), Spain

${ }^{50}$ Institut für Hochenergiephysik, Österr. Akad. d. Wissensch., Nikolsdorfergasse 18, A-1050 Vienna, Austria

${ }^{51}$ Inst. Nuclear Studies and University of Warsaw, Ul. Hoza 69, PL-00681 Warsaw, Poland

${ }^{52}$ Fachbereich Physik, University of Wuppertal, Postfach 100 127, D-5600 Wuppertal 1, Germany
} 


\section{Introduction}

During the last two years accurate measurements of the running coupling constant, $\alpha_{S}$, of quantum chromodynamics at $\mathrm{e}^{+} \mathrm{e}^{-}$colliders have been obtained from the analysis of event shape distributions measured in hadronic $Z^{0}$ decays $[1-3]$. In these publications, distributions in infrared and collinear safe observables are compared to QCD predictions in fixed order perturbation theory. The dominant uncertainty in the measurement of $\alpha_{S}$ results from the renormalization scale dependence of the coefficient of the second order term in $\alpha_{S}$.

Recently a resummation of leading and next-to-leading logarithmic contributions (NLLA) has been performed to all orders of perturbation theory for the distributions in thrust, heavy jet masses and jet broadening variables, and for the back-to-back energyenergy correlation [4-7]. In consequence the uncertainty of $\alpha_{S}$ due to the renormalization scale is reduced. Also the quantitative comparison between data and theory can be extended towards the 2-jet region, where gluons are emitted close to the directions of the produced quarks (infrared limit) and where the statistics are high. For some of the distributions studied in this paper, the resummed expressions have been applied to LEP data $[2,8,9]$.

In the following analysis $\alpha_{S}$ is determined using two methods. Method 1 concentrates on the 2-jet region where the cross-section can be reliably expressed as a power series in $\alpha_{S}$ containing only the leading and next-to-leading contributions of large logarithms. Values of $\alpha_{S}$ are determined from the distributions in thrust, heavy jet mass and jet broadening variables. Method 2 extends the comparison with theory into the region dominated by 3 -jet events. Here the resummed expression has to be matched with the full $\mathcal{O}\left(\alpha_{S}^{2}\right)$ expression for the different distributions. The corresponding $\alpha_{S}$ values, including the one from the analysis of the energy-energy correlation, are compared to the results of method 1.

In this paper section 2 briefly describes the DELPHI detector while section 3 introduces the relevant event shape variables and describes the data selection and corrections. In section 4 the theoretical expressions used to determine $\alpha_{S}$ are evaluated. Section 5 describes the hadronization correction. Also the sources of different systematic uncertainties and fit results are discussed, and finally the procedure to obtain a combined result for $\alpha_{S}$ including the correlation of the individual results is presented.

\section{The Detector}

A detailed description of the DELPHI apparatus has been presented in reference [10]. Here the components relevant for this analysis are briefly described.

The main part of the tracking system is a $2.7 \mathrm{~m}$ long Time Projection Chamber $(T P C)$ which measures the tracks of charged particles with a resolution of about $250 \mu \mathrm{m}$ in the $R \phi$-projection (transverse to the beam direction) and $0.9 \mathrm{~mm}$ along the $z$ direction (beam direction). The space between the $T P C$ and the beam pipe contains the Inner Detector $(I D)$ and a silicon microstrip Vertex Detector $(V D)$. Each $15^{\circ}$ sector of the $I D$ consists of a 24 wire jet chamber surrounded by a 5 layer proportional chamber. The Vertex Detector is built from three concentric shells of 24 silicon microstrip detector modules each $24 \mathrm{~cm}$ long. In the barrel region (polar angle $\theta$ relative to the beam axis between $43^{\circ}$ and $137^{\circ}$ ) the quality of tracking is further improved by the Outer Detector containing 5 layers of drift tubes. Each layer measures the $R \phi$-coordinate with a resolution of about $110 \mu \mathrm{m}$. Three layers also provide an approximate $z$ measurement. 
In the forward and backward regions ( $\theta$ in the range $11-33^{\circ}$ or $\left.147-169^{\circ}\right)$ two additional drift chamber systems improve the tracking. Forward chamber $A(F C A)$ consists of three pairs of wire planes rotated by $120^{\circ}$ with respect to each other, in order to resolve ambiguities internally. Forward chamber $B(F C B)$ consists of 12 wire planes twice repeating the orientation of $F C A$ and positioned directly in front of the forward electromagnetic calorimeter ( FEMC).

Electromagnetic clusters are measured in the barrel region $\left(\theta\right.$ between $43^{\circ}$ and $\left.137^{\circ}\right)$ by a High Density Projection Chamber $(H P C)$, and in the forward region ( $\theta$ in the range $10-36.5^{\circ}$ or $143.5-170^{\circ}$ ) by the $F E M C$, a matrix of 4522 lead glass blocks. The $H P C$ consists of 144 modules arranged in 6 rings around the beam axis. It is finely grained in 3 dimensions and thus allows a good two-shower separation and some particle identification from the shower shape.

\section{Data Analysis and Corrections}

For this analysis charged particles were used if they fulfilled the following criteria:

- momentum larger than $0.4 \mathrm{GeV} / \mathrm{c}$;

- over $30 \mathrm{~cm}$ measured track length;

- polar angle $\theta$ between $20^{\circ}$ and $160^{\circ}$;

- projection of impact parameter relative to the fitted main vertex below $4 \mathrm{~cm}$ in the plane transverse to the beam direction, and below $10 \mathrm{~cm}$ along the beam direction.

Photon showers were included if they were detected in the $H P C$ or the FEMC with an energy larger than $0.4 \mathrm{GeV}$ and if their polar angle was between $20^{\circ}$ and $160^{\circ}$.

Hadronic events were selected by requiring 5 or more charged particles, a total energy of all selected charged particles larger than $12 \%$ of the centre-of-mass energy, and larger than $3 \%$ in each hemisphere. To ensure that the event was well contained in the detector it was required that the angle between the sphericity axis and the beam axis exceeded $40^{\circ}$. A total of 177925 events survived these selections for the 1991 data.

All distributions have been obtained from the analysis of charged and neutral particles except for the distribution of the back-to-back energy-energy correlation ( $E E C$ ) where only charged particles were used. In order to correct the measured distributions for detector effects such as losses of charged and neutral particles, measurement errors and secondary interactions, events were generated with the JETSET 7.3 parton shower program [11] combined with the DYMU3 event generator [12] to simulate initial state photon radiation $(Q E D)$, and followed by a detailed simulation of the detector (DELSIM) [13]. The simulated data were processed in the same way as the real data. For a quantity $X$ the correction factor $C_{X}$ was calculated bin-by-bin as :

$$
C_{X}=\frac{\left(\frac{d \sigma}{d X}\right)_{\text {generated }}^{\text {DELSIM }}}{\left(\frac{d \sigma}{d X}\right)_{\text {reconstructed }}^{\text {DELSIM }}} \cdot \frac{\left(\frac{d \sigma}{d X}\right)^{\text {noQED }}}{\left(\frac{d \sigma}{d X}\right)^{Q E D}}
$$

where 'generated' includes all particles with a lifetime larger than $10^{-9} \mathrm{~s}$ without detector simulation, and 'reconstructed' indicates the full detector simulation and reconstruction of the simulated raw data.

In this analysis experimental distributions for six quantities are presented, namely for thrust $T$, two different definitions of normalized heavy jet mass squared $\rho$, two jet broadening measures $B, B_{W}$ and back-to-back energy-energy correlation $E E C$. For these distributions leading and next-to-leading logarithms have been resummed to all orders of 
$\alpha_{S}$ in references [4-7]. The definitions of these event shape variables are :

$$
\begin{gathered}
T=\max _{\vec{n}_{t h r}} \frac{\sum_{i}\left|\vec{p}_{i} \cdot \vec{n}_{t h r}\right|}{\sum_{i}\left|\vec{p}_{i}\right|} \\
\rho^{(T)}=\frac{1}{E_{v i s}^{2}} \cdot \max \left[\left(\sum_{\vec{p}_{i} \cdot \vec{n}_{t h r}>0} p_{i}\right)^{2},\left(\sum_{\vec{p}_{i} \cdot \vec{n}_{t h r}<0} p_{i}\right)^{2}\right]
\end{gathered}
$$

where $i$ runs over all final state particles, $\vec{n}_{t h r}$ is the thrust axis, and $E_{v i s}$ the total energy of all visible particles with four-momentum $p_{i}$. In equation (3) charged particles are assumed to have the pion mass while neutrals are assumed to be massless.

Among all possible ways to divide the particle system into two groups $a$ and $b$ the one which minimizes the sum of the squared invariant masses $M_{a}^{2}+M_{b}^{2}$ was selected in order to get the alternative jet mass $\rho^{(M)}[14]$ :

$$
\rho^{(M)}=\frac{1}{E_{v i s}^{2}} \cdot \max \left(M_{a}^{2}, M_{b}^{2}\right)
$$

In reference [7] two new $\mathrm{e}^{+} \mathrm{e}^{-}$event shape variables were introduced which measure the jet broadening by summing up the transverse momenta of the final state particles relative to the thrust axis. The total jet broadening $B$ and wide jet broadening $B_{W}$ are defined as follows:

$$
\begin{gathered}
B=B_{+}+B_{-} \\
B_{W}=\max \left(B_{+}, B_{-}\right)
\end{gathered}
$$

with

$$
B_{ \pm}=\frac{ \pm \vec{p}_{i} \cdot \vec{n}_{t h r}>0}{2 \sum_{i}\left|\vec{p}_{i}\right|}
$$

where $i$ again runs over all final state particles.

The back-to-back energy-energy correlation is defined from the distribution of angles $\chi$ between all particle pairs in an event weighted by their energies :

$$
\operatorname{EEC}(\chi)=\frac{1}{N} \frac{1}{\Delta \chi} \sum_{\text {events }}^{N} \sum_{i, j}^{N_{\text {particles }}} \frac{E_{i} E_{j}}{E_{v i s}^{2}} \int_{\chi-\frac{\Delta \chi}{2}}^{\chi+\frac{\Delta \chi}{2}} \delta\left(\chi-\chi_{i j}\right) d \chi
$$

where $\chi$ is taken larger than $90^{\circ}$ and $\Delta \chi$ is the bin width of the histogram.

\section{QCD Predictions in NLLA}

Pure second order QCD predictions can describe the distribution of an infrared and collinear safe shape variable only in a limited kinematic region dominated by the 3 -jet rate, where higher order effects are negligible. On the other hand in the 2-jet region higher order terms grow in the case of exponentiation [15] like $\ln ^{n+1} y \alpha_{S}^{n}$ as the shape variable $y$ is close to its infrared limit $y \rightarrow 0$. Therefore in this region any fixed order expansion of the cross-section must fail. To extend the range towards the infrared limit it is essential to calculate at least the leading higher order terms. The expansion of the integrated cross-section

$$
R\left(\tilde{y}, \alpha_{S}\right)=\frac{1}{\sigma_{t o t}} \sigma(y<\tilde{y})
$$


at the scale $Q^{2} \equiv s$ can be written in the form [4-7]:

$$
\begin{array}{r}
c R\left(y, \alpha_{S}\left(Q^{2}\right)\right)=\left(1+C_{1} \alpha_{S}\left(Q^{2}\right)+C_{2} \alpha_{S}^{2}\left(Q^{2}\right)+\ldots\right) \cdot \Sigma\left(L, \alpha_{S}\left(Q^{2}\right)\right) \\
+F_{1}(y) \alpha_{S}\left(Q^{2}\right)+F_{2}(y) \alpha_{S}^{2}\left(Q^{2}\right)+\ldots
\end{array}
$$

with

$$
\begin{aligned}
& L=\ln y \\
& c=\left\{\begin{array}{lll}
1 & : & T, \rho^{(T)}, \rho^{(M)}, B, B_{W} \\
2 & : & \text { back-to-back } E E C
\end{array}\right.
\end{aligned}
$$

where the $C_{i}$ are constant and the $F_{i}(y)$ vanish in the infrared limit $y \rightarrow 0$. Here $y$ stands for $(1-T), \rho^{(T)}, \rho^{(M)}, B, B_{W}$ or $(1+\cos \chi) / 2$, respectively. All logarithmic divergencies can be included in the function $\Sigma\left(L, \alpha_{S}\left(Q^{2}\right)\right)$. In the case of exponentiation which holds for thrust, heavy jet masses, jet broadening variables and back-to-back energy-energy correlation, the logarithm of the function $\Sigma$ can be written in the form :

$$
\begin{aligned}
\ln \left(\Sigma\left(L, \alpha_{S}\right)\right) & =\left(G_{12} L^{2}+G_{11} L\right) \\
& +\left(G_{23} L^{3}+G_{22} L^{2}+\alpha_{S}\right. \\
& +\left(G_{34} L^{4}+G_{33} L^{3}+\right. \\
& +\underbrace{\ldots}_{L L} \underbrace{}_{N L L}
\end{aligned}
$$

Once exponentiation is established the leading and next-to-leading terms, denoted in equation (13) as $L L$ and $N L L$, can be resummed to all orders of $\alpha_{S}$. Logarithms weaker than next-to-leading, such as the $\left(G_{21} L\right) \alpha_{S}^{2}$ term, are called subleading. At present subleading terms higher than $\mathcal{O}\left(\alpha_{S}^{2}\right)$ remain uncalculated and have to be estimated or neglected. A transition from the scale $Q^{2}$ to a scale $\mu^{2}$ induces a $f=\mu^{2} / Q^{2}$ dependence of the next-to-leading terms, i. e. $\ln \Sigma$ explicitly depends on $f$.

Apart from the combination of the second order calculation and the resummed logarithms as suggested in equation (10), alternative matching schemes have been proposed $[8,16]$, which differ in terms of the order $\mathcal{O}\left(\alpha_{S}^{3} \ln ^{2} y\right)$. If the first and second order coefficients $A$ and $B$, which can be taken from reference [14] or computed by using the EVENT program $[14,17]$, are defined through

$$
c R\left(y, \alpha_{S}\right)=1+A(y) \alpha_{S}+B(y) \alpha_{S}^{2}
$$

and the functions $g_{1}$ and $g_{2}$ as

$$
\begin{aligned}
& g_{1}(L)=G_{12} L^{2}+G_{11} L \\
& g_{2}(L)=G_{23} L^{3}+G_{22} L^{2}+G_{21} L
\end{aligned}
$$

the following matching schemes can be defined :

ln $R$-matching scheme : This scheme follows from references [4,5] :

$$
\ln c R\left(y, \alpha_{S}\right)=\ln \left(\Sigma\left(L, \alpha_{S}\right)\right)+H_{1}(y) \alpha_{S}+H_{2}(y) \alpha_{S}^{2}
$$

with

$$
\begin{aligned}
& H_{1}(y)=A(y)-g_{1}(L) \\
& H_{2}(y)=B(y)-\frac{1}{2} A^{2}(y)-g_{2}(L)
\end{aligned}
$$

$R-G_{21}$-matching scheme : This scheme follows from equation (10) and is suggested in reference $[6]$ :

$$
c R\left(y, \alpha_{S}\right)=\left(1+C_{1} \alpha_{S}+C_{2} \alpha_{S}^{2}\right) \Sigma\left(L, \alpha_{S}\right)+F_{1}(y) \alpha_{S}+F_{2}(y) \alpha_{S}^{2}
$$


with

$$
\begin{aligned}
& F_{1}(y)=A(y)-g_{1}(L)-C_{1} \\
& F_{2}(y)=B(y)-\frac{1}{2} g_{1}^{2}(L)-g_{2}(L)-C_{1} g_{1}(L)-C_{2}
\end{aligned}
$$

where the coefficient $G_{21}$ is known from the full second order calculation [14,18].

$R$-matching scheme : This scheme also follows from equation (10), but now with $g_{2}$ and $\ln \Sigma$ in NLLA only, i. e. without the $G_{21} L$ term.

In this analysis the ambiguity introduced by these different matching schemes is used for evaluating one source of theoretical uncertainties. From a theoretical point of view the $\ln R$ - and $R-G_{21}$-schemes are preferred, since in both schemes all known logarithms are exponentiated, while the $R$-matching suffers from the fact that the known logarithmic term $G_{21} L$ is only included in second order and not exponentiated. This leads to large differences between the $\ln R$ - or $R$ - $G_{21}$-scheme and the $R$-scheme if this term is large. Nevertheless the $R$-scheme can be used in a restricted fit range to estimate the influence of uncalculated terms. This is discussed in more detail in the next section.

When combining second order theory with resummed logarithms one has to take into account that the resummed terms do not vanish at the upper kinematic boundary. Therefore the resummed logarithms are redefined in the following form [16] :

$$
L=\ln \left(1 / y-1 / y_{\max }+1\right)
$$

Here $y_{\max }$ is the upper kinematic boundary for each variable and is listed in Table 7.

As a consequence of the explicit inclusion of higher order terms in the theoretical prediction a reduced scale dependence is expected. The small scales found in former pure second order $\alpha_{S}$ measurements [2,3], which were necessary to compensate unknown higher order terms, are not needed in the resummed calculation and are even forbidden, since such small scales introduce additional terms of the type $\ln ^{n} f$ to all orders of $\alpha_{S}$. This would lead to some double counting of the higher order terms.

\section{Confrontation of QCD in NLLA and Data}

Before theoretical expressions describing parton distributions can be compared with the experimental data, corrections must be made for hadronization effects, i.e. effects resulting from the transition of the parton state into the observable hadronic state. For the global event shape variables, thrust, jet broadening and heavy jet masses, it is assumed that hadronization effects can be described by a correlation matrix $C$ which connects the parton state with the hadronic final state:

$$
\left.\frac{\Delta \sigma}{\Delta y_{i}}\right|_{\text {Hadron }}=\left.\sum_{j} C_{i j} \frac{\Delta \sigma}{\Delta y_{j}}\right|_{\text {Parton }}
$$

In the case of the back-to-back energy-energy correlation, which is defined for each pair of charged particles, hadronization effects are described by a linear bin-by-bin correction vector $C_{i}$ :

$$
\left.\frac{\Delta R\left(\chi_{i}\right)}{\Delta \chi_{i}}\right|_{\text {Hadron }}=\left.C_{i} \frac{\Delta R\left(\chi_{i}\right)}{\Delta \chi_{i}}\right|_{\text {Parton }}
$$

These corrections were determined using 1000000 Monte Carlo events produced with JETSET 7.3 tuned to the DELPHI data [19]. In addition to this Monte Carlo sample, different tunings and models were used for the hadronization correction yielding an estimate of the systematic uncertainty of this correction, as detailed later. 
For the confrontation of $Q C D$ with data and the determination of $\alpha_{S}$ two approaches have been used :

1. Following the suggestion of references $[7,20]$ to consider two distinct theories, each applicable in certain kinematic regions (namely the resummed leading and next-toleading logarithms ( NLLA) on one side, and pure $\mathcal{O}\left(\alpha_{S}^{2}\right)$ theory on the other side), $\alpha_{S}$ is extracted from one measured bin in the extreme 2 -jet region, where $y$ is small, using pure NLLA without combination with second order theory. Figure 1a shows the ratio $\omega$ of the resummed logarithms (first two columns of eq. (13), denoted as $\left.\ln \Sigma^{(N L L A)}\right)$ and the non-exponentiating second order contributions :

$$
\omega=\frac{\ln \Sigma^{(N L L A)}}{H_{1} \alpha_{S}+H_{2} \alpha_{S}^{2}}
$$

In the extreme 2-jet region where the ratio $\omega$ becomes large, the theory should be completely dominated by the resummed logarithms signalling the reliability of this approach. This method, which will be denoted as method 1, is complementary to pure second order $\alpha_{S}$ determinations using data in the 3 -jet region. It offers the possibility of comparing a theory which is known to all orders of $\alpha_{S}$ in $N L L A$ with the data. However, this approach is not applicable to the back-to-back energyenergy correlation due to the fact that here the ratio $\omega$ (Figure $2 \mathrm{a}$ ) does not become sufficiently large in the accessible range, which is limited towards small $y$-values by the pole in the resummed next-to-leading logarithms as visible in Figure $2 b$ [6].

2. While the first method aims to measure $\alpha_{S}$ to all orders in NLLA, the second method tests a combined $N L L A$ and $\mathcal{O}\left(\alpha_{S}^{2}\right)$ theory over a wide kinematical range including the 3 -jet region. Therefore expressions (24-25) are confronted with the experimental distributions by means of a least square fit supplying a measurement of $\Lambda_{\overline{M S}}$ which can be translated into $\alpha_{S}\left(M_{\mathrm{Z}}^{2}\right)$. For the fit only statistical errors are considered. Figures 3 to 8 show the hadron data compared with the fit result of the $\ln R$-matching scheme at the renormalization scale $\mu^{2}=Q^{2} \equiv s$. The fit range is chosen in such a way that:

- Acceptance corrections are below about $20 \%$ and hadronization corrections below about $40 \%$.

- One keeps away from the pole of the ratio of the next-to-leading logarithms and the leading logarithms (Figure $1 \mathrm{~b}$ and $2 \mathrm{~b}$ ) at small $y$ values. Otherwise subleading terms cannot be neglected.

- The data can be well described by the theoretical prediction.

These criteria lead to the following fit ranges

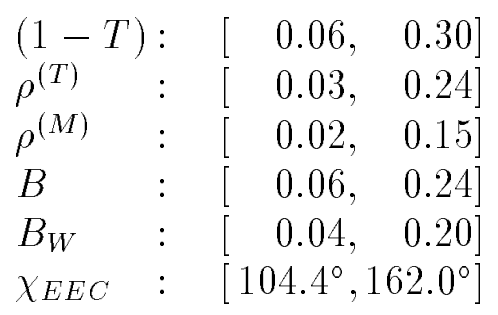

For all variables the fit ranges have been extended towards the infrared limit in comparison to the more restricted fit ranges used for the determination of $\alpha_{S}$ in $\mathcal{O}\left(\alpha_{S}^{2}\right)[3]$. Below the lower limit of the fit range, hadronization corrections increase strongly for $1-T, \rho^{(T)}, \rho^{(M)}, B$ and $B_{W}$. For the $R$-scheme, which is used in this analysis only to estimate the theoretical uncertainty, a fit range restricted to the 
3 -jet region is used. This is necessary due to the one logarithmic divergent term $G_{21} L$, which is not exponentiated in the $R$-scheme and so leads to an unphysical divergency of the predicted cross-section in the 2-jet limit. The size of this term can be seen from Figures $1 \mathrm{c}$ and $2 \mathrm{c}$, where the ratio $G_{21} \ln y / G_{22} \ln ^{2} y$ is plotted for thrust and for back-to-back energy-energy correlation. This ratio is one order of magnitude larger for $E E C$ than for thrust, indicating the large matching scheme ambiguity of the EEC.

The data can be described well by the combined theory over a wide kinematic region. Note that the value of $\chi^{2} / N D F$ is evaluated from statistical errors only. These are very small, in particular in the case of the $E E C$.

\subsection{Systematic Uncertainties}

To estimate the systematic error of $\alpha_{S}$, three sources of uncertainties are investigated:

1. Experimental uncertainty : Several data samples with different selections were used to calculate the acceptance corrections and to estimate all other experimental uncertainties. In addition an analysis using only charged particles was performed with events selected to be fully contained in the barrel part of DELPHI. The bin-by-bin systematic experimental errors are quoted in Tables 1 to 6 .

2. Hadronization correction : In addition to the JETSET 7.3 Monte Carlo sample, yielding the central value of the analysis, the HERWIG 5.4 and ARIADNE 3.1 models tuned to LEP data [21] were used to evaluate the hadronization correction. All three models differ in parton state generation. Although JETSET and HERWIG both follow the parton shower model, they differ in some details :

- JETSET includes the full $\mathcal{O}\left(\alpha_{S}\right)$ matrix element for hard gluon radiation,

- HERWIG takes into account interference phenomena within the shower to full leading order.

While JETSET and HERWIG formulate the QCD shower in terms of partons, ARIADNE uses the colour dipole formalism. For fragmentation JETSET and ARIADNE both use the JETSET string fragmentation, while HERWIG incorporates cluster fragmentation.

To estimate the uncertainty originating from the parameter tuning, two alternative tunings of JETSET, performed by the DELPHI collaboration, were used in addition. Furthermore the parton virtuality $Q_{0}=1 \mathrm{GeV}$ which limits the shower evolution in JETSET, was varied between $Q_{0}=0.2$ and $5.0 \mathrm{GeV}$. The systematic hadronization error is then estimated as the variance of the fitted $\alpha_{S}$ values obtained by using all hadronization corrections as mentioned above.

3. Theoretical uncertainties : To estimate the theoretical uncertainties, fits at different values of $\mu^{2} / Q^{2}$ ranging from 0.5 to 2.0 were performed. In addition for method 1 the bin selected for the determination of $\alpha_{S}$ was slightly shifted towards the 3-jet region. For method 2, two different fit ranges were used for each variable. One of these fit ranges was shifted towards the 2-jet region and the other towards the 3-jet region. A further uncertainty of method 2 originates from the selection of the matching scheme, and was estimated by evaluating $\alpha_{S}$ with the $R$-scheme instead of the $\ln R$-scheme. The variance of the $\alpha_{S}$ values obtained yields an estimate for the theoretical uncertainty.

\subsection{Results}

Table 8 presents the bin edges and bin contents used for method 1 and the results obtained at scale $\mu^{2}=M_{\mathrm{Z}}^{2}$. For each variable the bin selected for the evaluation of $\alpha_{S}$ 
is chosen in such a way, that the ratio plotted in Figure 1a becomes as large as allowed by the data. Here larger hadronization and acceptance corrections are accepted than for the fit range of method 2 .

The results found with method 2 from $\chi^{2}$ fits to the particular variables at the scale $\mu^{2}=M_{\mathrm{Z}}^{2}$ using the different matching schemes as defined in section 4 , are summarized in Table 9. The errors quoted there are the full experimental and theoretical errors evaluated as described in section 5.1. The fits in the $\ln R$ - and $R$ - $G_{21}$-schemes are performed over the full range including the 2 -jet region, while for the fits in the $R$-scheme a range restricted to the 3 -jet region is used as detailed in section 5 . The values found with the $\ln R$ - and the $R-G_{21}$-schemes agree well. Regarding the larger difference between the results from these two schemes and the $R$-scheme one has also to note the different fit range used for the fits in the latter scheme. The results of method 1 (Table 8) and those of method 2 (Table 9 ) agree if the $\ln R$ - or the $R-G_{21}$-scheme are used in method 2. Due to the unphysical divergency of the $R$-scheme the $\ln R$-scheme is chosen to be the central matching scheme in method 2. This scheme is preferred also in references [4,5]. The upper plot of Figure 9 shows the dependence of $\alpha_{S}$ on the scale $\mu^{2} / Q^{2}$ of each particular variable investigated in method 2. With the exception of the $E E C$ and the wide jet broadening $B_{W}$, all variables agree well and behave similarly.

\subsection{Combined result}

To combine the results from the fits to the individual variables, statistical correlations are taken into account. Therefore a covariance matrix $\hat{\sigma}$, which itself is the sum of four matrices, is calculated:

$$
\hat{\sigma}=\hat{\sigma}^{(\text {stat })}+\hat{\sigma}^{(e x p)}+\hat{\sigma}^{(h a d r)}+\hat{\sigma}^{(t h e o)}
$$

$\hat{\sigma}^{(\text {stat })}$ is obtained by splitting the full data sample in 10 subsamples. To each subsample and each variable a fit is performed yielding $10 \times 6$ values $\alpha_{S(i k)}, i=1, \ldots, 6 ; k=1, \ldots, 10$, from which, $\hat{\sigma}^{(\text {stat })}$ is computed:

$$
\hat{\sigma}_{i j}^{(s t a t)}=\frac{1}{N(N-1)} \sum_{k=1}^{N=10}\left(\alpha_{S(i k)}-\bar{\alpha}_{S(i)}\right)\left(\alpha_{S(j k)}-\bar{\alpha}_{S(j)}\right)
$$

with

$$
\bar{\alpha}_{S(i)}=\frac{1}{N} \sum_{k=1}^{N=10} \alpha_{S(i k)}
$$

$\hat{\sigma}^{(e x p)}, \hat{\sigma}^{(h a d r)}$ and $\hat{\sigma}^{(t h e o)}$ are obtained similarly but now with the $\alpha_{S}$ values obtained in the estimation of the experimental, hadronization and theoretical systematic uncertainties, respectively, instead of the values obtained from the data subsamples. Since correlations of systematic uncertainties are hard to work out reliably, in these latter categories the nondiagonal covariances are set to zero. Once the covariance matrix $\hat{\sigma}$ has been calculated, a combined average value for $\alpha_{S}$ and the errors can be computed.

The combined result obtained at the scale $\mu^{2}=M_{\mathrm{Z}}^{2}$ with analysis method 1 is :

$$
\alpha_{S}=0.118 \pm 0.002(\text { stat }+\exp ) \pm 0.003(\text { hadr }) \pm 0.006(\text { theo })
$$

The first error quoted is the experimental uncertainty, which includes the statistical error and the uncertainty of the detector simulation. The second error is due to hadronization correction and the third error is an estimate of all theoretical uncertainties. 
For method 2, combined $\alpha_{S}$-values using the $\ln R$-scheme are quoted for several values of the renormalization scale, as shown in the lower plot of Figure 9. The band corresponds to the total error of the combined values. Since the correlation matrices are expected to be scale independent, they were calculated only at the scale $\mu^{2}=M_{\mathrm{Z}}^{2}$ but applied to all investigated renormalization scales.

The value obtained with analysis method 2 at $\mu^{2}=M_{Z}^{2}$ is :

$$
\alpha_{S}=0.123 \pm 0.002(\text { stat }+\exp ) \pm 0.002(\text { hadr })
$$

where the errors are again the combined statistical and experimental error, and the uncertainty due to hadronization correction. From the remaining scale dependence (estimated using the range $\left.0.5 \leq \mu^{2} / Q^{2} \leq 2.0\right)$ and matching ambiguity, the theoretical uncertainty of method 2 is estimated to :

$$
\Delta \alpha_{S}= \pm 0.005 \text { (scale+matching ambiguity) }
$$

It should be pointed out that this theoretical error is estimated as an "one standard deviation error". The uncertainty due to a certain arbitrariness in the choice of the fit range is estimated to :

$$
\Delta \alpha_{S}= \pm 0.002 \text { (fit range) }
$$

This yields a total theoretical error of :

$$
\Delta \alpha_{S}= \pm 0.005
$$

\section{Conclusions}

From $178000 \mathrm{Z}^{0}$ hadronic decays collected in 1991 with the DELPHI detector at the LEP collider, a new measurement of the strong coupling constant $\alpha_{S}$ has been performed, using the next-to-leading logarithm approximation (NLLA) of $Q C D$.

Using theoretical expressions including resummation of leading and next-to-leading logarithms to all orders of $\alpha_{S}$, which describe parton distributions for thrust, heavy jet mass, jet broadening and back-to-back energy-energy correlation, $\alpha_{S}$ was determined following two different approaches. The first method concentrates on the extreme 2-jet region where the resummed logarithms dominate, and is in some sense complementary to a pure second order $\alpha_{S}$ determination which is restricted to the 3 -jet region. This method can only be applied to thrust, the heavy jet mass and the jet broadening measures.

The second method confronts the combined $N L L A$ and $\mathcal{O}\left(\alpha_{S}^{2}\right)$ calculation with data. The values of $\alpha_{S}$ obtained from both methods agree within errors if the $\ln R$ - or $R-G_{21}$ matching schemes are used for method 2. While the first approach should be reliable to determine $\alpha_{S}$ only from the 2-jet region, the second tests whether a combined calculation is able to describe the experimental distributions over a wide kinematical range including the 3 -jet region. Therefore the result obtained with method 1 is considered as a consistency check, and the final result :

$$
\alpha_{S}=0.123 \pm 0.006 \text { at the scale } \mu^{2}=M_{\mathrm{Z}}^{2}
$$

is based on method 2 only.

If in method 2 the $R$ - $G_{21}$-scheme is chosen instead of the $\ln R$-scheme, the final value is $\alpha_{S}=0.122 \pm 0.006$. In a previous paper [3] based on $\mathcal{O}\left(\alpha_{S}^{2}\right)$ perturbation theory, a value of $\alpha_{S}\left(M_{Z}^{2}\right)=0.115 \pm 0.007$ was determined. This result was obtained from a combined analysis of eight distributions of event shape variables, where hadronization corrections were performed by using parton shower models. Within errors the results of the $\mathcal{O}\left(\alpha_{S}^{2}\right)$ and the NLLA analysis are consistent. 


\section{Acknowledgements}

We are greatly indebted to our technical collaborators and to the funding agencies for their support in building and operating the DELPHI detector, and to the members of the CERN-SL Division for the excellent performance of the LEP collider. We would like to thank S. Catani, G. Turnock and B.R. Webber for useful discussions.

\section{References}

[1] OPAL coll., M.Z. Akrawy et al.: Phys. Lett. B235 (1990) 389;

DELPHI coll., P. Abreu et al.: Phys. Lett. B247 (1990) 167;

L3 coll., B. Adeva et al.: Phys. Lett B248 (1990) 464;

MARK II coll., S. Komamiya et al.: Phys. Rev. Lett. 64 (1990) 987;

DELPHI coll., P. Abreu et al.: Phys. Lett. B252 (1990) 149;

OPAL coll., M.Z. Akrawy et al.: Phys. Lett. B252 (1990) 159;

OPAL coll., M.Z. Akrawy et al.: Z. Phys. C49 (1991) 375;

ALEPH coll., D. Decamp et al.: Phys. Lett. B255 (1991) 623;

L3 coll., B. Adeva et al.: Phys. Lett. B257 (1991) 469;

ALEPH coll., D. Decamp et al.: Phys. Lett. B257 (1991) 479;

OPAL coll., P.D. Acton et al.: Phys. Lett. B276 (1992) 547.

[2] OPAL coll., P.D. Acton et al.: Z. Phys. C55 (1992) 1.

[3] DELPHI coll., P. Abreu et al.: Z. Phys. C54 (1992) 55.

[4] S. Catani, G. Turnock, B.R. Webber and L. Trentadue: Phys. Lett. B263 (1991) 491.

[5] S. Catani, G. Turnock and B.R. Webber: Phys. Lett. B272 (1991) 368.

[6] G. Turnock: Preprint Cavendish-HEP-92/3 (1992).

[7] S. Catani, G. Turnock and B.R. Webber: Preprint CERN-TH. 6570/92 (1992).

[8] ALEPH coll., D. Decamp et al.: Phys. Lett. B284 (1992) 163.

[9] L3 coll., B. Adeva et al.: Phys. Lett. B284 (1992) 471.

[10] DELPHI coll., P. Aarnio et al.: Nucl. Inst. and Meth. A 303 (1991) 233.

[11] T. Sjöstrand: Comp. Phys. Com. 27 (1982) 243; ibid. 28 (1983) 229; T. Sjöstrand, M. Bengtsson: Comp. Phys. Com. 43 (1987) 367.

[12] J.E. Campagne, R. Zitoun: Z. Phys. C43 (1989) 469.

[13] DELSIM User Manual, DELPHI 87-96 PROG-99, Geneva (July 1989).

[14] Z. Kunszt, P. Nason: in "Z Physics at LEP 1", ed. G. Altarelli, R. Kleiss and Z. Verzegnassi, CERN 89-08, Geneva (1989), Vol. 1, p. 373.

[15] S. Catani: Preprint CERN-TH. 6281/91 (1991).

[16] B.R. Webber: "Jets in Perturbation Theory", Proceedings of the Workshop "QCD 20 years later", Aachen, June 9 - 13, 1992; and private communication.

[17] P. Nason: private communication.

[18] R. K. Ellis, D. A. Ross and A. E. Terrano: Nucl. Phys. B178 (1981) 421.

[19] W. de Boer et al.: Proceedings of the "Workshop on Detector and Event Simulation in High Energy Physics", Amsterdam, April 1991, p. 616, Eds. K. Bos and B. van Eijk.

[20] G. Turnock: in Proceedings of the Workshop "QCD 20 years later", Aachen, June 9 - 13, 1992; and private communication.

[21] OPAL coll., M. Z. Akrawy et al.: Z. Phys. C47 (1990) 505. 


\section{Tables}

\begin{tabular}{|c|c|}
\hline$T$ & $\frac{1}{\sigma_{+\circ t}} \frac{\Delta \sigma}{\Delta T} \pm($ stat $) \pm(\exp )$ \\
\hline 0.605 & $0.012 \pm 0.007 \pm 0.007$ \\
\hline 0.615 & $0.006 \pm 0.003 \pm 0.003$ \\
\hline 0.625 & $0.010 \pm 0.004 \pm 0.004$ \\
\hline 0.635 & $0.021 \pm 0.006 \pm 0.007$ \\
\hline 0.645 & $0.07 \pm 0.01 \pm 0.02$ \\
\hline 0.655 & $0.07 \pm 0.01 \pm 0.03$ \\
\hline 0.665 & $0.10 \pm 0.01 \pm 0.01$ \\
\hline 0.675 & $0.12 \pm 0.01 \pm 0.01$ \\
\hline 0.685 & $0.15 \pm 0.02 \pm 0.02$ \\
\hline 0.695 & $0.18 \pm 0.02 \pm 0.02$ \\
\hline 0.705 & $0.23 \pm 0.02 \pm 0.01$ \\
\hline 0.715 & $0.27 \pm 0.02 \pm 0.03$ \\
\hline 0.725 & $0.33 \pm 0.02 \pm 0.03$ \\
\hline 0.735 & $0.36 \pm 0.03 \pm 0.02$ \\
\hline 0.745 & $0.40 \pm 0.03 \pm 0.03$ \\
\hline 0.755 & $0.48 \pm 0.03 \pm 0.02$ \\
\hline 0.765 & $0.51 \pm 0.03 \pm 0.02$ \\
\hline 0.775 & $0.55 \pm 0.03 \pm 0.03$ \\
\hline 0.785 & $0.62 \pm 0.03 \pm 0.03$ \\
\hline 0.795 & $0.76 \pm 0.04 \pm 0.03$ \\
\hline 0.805 & $0.77 \pm 0.04 \pm 0.02$ \\
\hline 0.815 & $0.89 \pm 0.04 \pm 0.04$ \\
\hline 0.825 & $1.07 \pm 0.04 \pm 0.03$ \\
\hline 0.835 & $1.13 \pm 0.04 \pm 0.05$ \\
\hline 0.845 & $1.32 \pm 0.05 \pm 0.03$ \\
\hline 0.855 & $1.42 \pm 0.05 \pm 0.03$ \\
\hline 0.865 & $1.71 \pm 0.06 \pm 0.05$ \\
\hline 0.875 & $1.92 \pm 0.06 \pm 0.03$ \\
\hline 0.885 & $2.28 \pm 0.06 \pm 0.04$ \\
\hline 0.895 & $2.71 \pm 0.07 \pm 0.11$ \\
\hline 0.905 & $2.99 \pm 0.07 \pm 0.06$ \\
\hline 0.915 & $3.90 \pm 0.08 \pm 0.13$ \\
\hline 0.925 & $4.62 \pm 0.09 \pm 0.11$ \\
\hline 0.935 & $5.7 \pm 0.1 \pm 0.1$ \\
\hline 0.945 & $7.4 \pm 0.1 \pm 0.2$ \\
\hline 0.955 & $9.7 \pm 0.1 \pm 0.4$ \\
\hline 0.965 & $13.6 \pm 0.2 \pm 0.2$ \\
\hline 0.975 & $16.2 \pm 0.2 \pm 0.8$ \\
\hline 0.985 & $10.1 \pm 0.1 \pm 0.5$ \\
\hline 0.995 & $1.40 \pm 0.04 \pm 0.14$ \\
\hline
\end{tabular}

Table 1: Thrust distribution corrected for detector acceptance and initial state photon radiation. 


\begin{tabular}{|c||cc|}
\hline$\rho^{(T)}$ & $\frac{1}{\sigma_{\text {tot }}} \frac{\Delta \sigma}{\Delta \rho(T)} \pm(\mathrm{stat}) \pm(\mathrm{exp})$ \\
\hline 0.005 & 1.84 & $\pm 0.04 \pm 0.58$ \\
\hline 0.015 & 15.2 & $\pm 0.1 \pm 1.3$ \\
\hline 0.025 & 20.2 & $\pm 0.2 \pm 0.5$ \\
\hline 0.035 & 14.1 & $\pm 0.2 \pm 0.8$ \\
\hline 0.045 & 9.9 & $\pm 0.1 \pm 0.5$ \\
\hline 0.055 & 7.1 & $\pm 0.1 \pm 0.4$ \\
\hline 0.065 & 5.5 & $\pm 0.1 \pm 0.3$ \\
\hline 0.075 & 4.30 & $\pm 0.09 \pm 0.22$ \\
\hline 0.085 & 3.47 & $\pm 0.08 \pm 0.17$ \\
\hline 0.095 & 2.78 & $\pm 0.07 \pm 0.16$ \\
\hline 0.105 & 2.30 & $\pm 0.07 \pm 0.12$ \\
\hline 0.115 & 1.99 & $\pm 0.06 \pm 0.09$ \\
\hline 0.125 & 1.69 & $\pm 0.06 \pm 0.05$ \\
\hline 0.135 & 1.37 & $\pm 0.05 \pm 0.05$ \\
\hline 0.145 & 1.18 & $\pm 0.05 \pm 0.07$ \\
\hline 0.155 & 1.05 & $\pm 0.05 \pm 0.06$ \\
\hline 0.165 & 0.89 & $\pm 0.04 \pm 0.10$ \\
\hline 0.175 & 0.77 & $\pm 0.04 \pm 0.06$ \\
\hline 0.185 & 0.67 & $\pm 0.04 \pm 0.02$ \\
\hline 0.195 & 0.54 & $\pm 0.03 \pm 0.03$ \\
\hline 0.205 & 0.47 & $\pm 0.03 \pm 0.04$ \\
\hline 0.215 & 0.39 & $\pm 0.03 \pm 0.02$ \\
\hline 0.225 & 0.34 & $\pm 0.03 \pm 0.03$ \\
\hline 0.235 & 0.29 & $\pm 0.02 \pm 0.02$ \\
\hline 0.245 & 0.23 & $\pm 0.02 \pm 0.02$ \\
\hline 0.255 & 0.18 & $\pm 0.02 \pm 0.02$ \\
\hline 0.265 & 0.166 & $\pm 0.017 \pm 0.006$ \\
\hline 0.275 & 0.13 & $\pm 0.01 \pm 0.01$ \\
\hline 0.285 & $0.088 \pm 0.012 \pm 0.006$ \\
\hline 0.295 & $0.062 \pm 0.009 \pm 0.003$ \\
\hline 0.305 & 0.048 & $\pm 0.008 \pm 0.012$ \\
\hline 0.315 & 0.035 & $\pm 0.007 \pm 0.005$ \\
\hline 0.325 & 0.024 & $\pm 0.006 \pm 0.007$ \\
\hline 0.335 & $0.022 \pm 0.006 \pm 0.003$ \\
\hline 0.345 & $0.011 \pm 0.003 \pm 0.002$ \\
\hline 0.355 & 0.007 & $\pm 0.003 \pm 0.003$ \\
\hline 0.365 & $0.006 \pm 0.003 \pm 0.001$ \\
\hline 0.375 & $0.002 \pm 0.002 \pm 0.002$ \\
\hline 0.385 & 0.002 & $\pm 0.002 \pm 0.001$ \\
\hline 0.395 & $0.0014 \pm 0.0015 \pm 0.0007$ \\
\hline
\end{tabular}

Table 2: Heavy jet mass distribution (thrust definition) corrected for detector acceptance and initial state photon radiation. 


\begin{tabular}{|l||cc|}
\hline$\rho^{(M)}$ & $\frac{1}{\sigma_{\text {tot }}} \frac{\Delta \sigma}{\Delta \rho^{(M)}} \pm($ stat $) \pm(\exp )$ \\
\hline 0.005 & 1.87 & $\pm 0.05 \pm 0.58$ \\
\hline 0.015 & 15.5 & $\pm 0.2 \pm 1.4$ \\
\hline 0.025 & 20.6 & $\pm 0.2 \pm 0.7$ \\
\hline 0.035 & 14.4 & $\pm 0.2 \pm 0.9$ \\
\hline 0.045 & 10.0 & $\pm 0.1 \pm 0.6$ \\
\hline 0.055 & 7.2 & $\pm 0.1 \pm 0.4$ \\
\hline 0.065 & 5.5 & $\pm 0.1 \pm 0.2$ \\
\hline 0.075 & 4.34 & $\pm 0.09 \pm 0.29$ \\
\hline 0.085 & 3.54 & $\pm 0.08 \pm 0.20$ \\
\hline 0.095 & 2.90 & $\pm 0.07 \pm 0.12$ \\
\hline 0.105 & 2.40 & $\pm 0.07 \pm 0.12$ \\
\hline 0.115 & 2.09 & $\pm 0.06 \pm 0.08$ \\
\hline 0.125 & 1.70 & $\pm 0.06 \pm 0.12$ \\
\hline 0.135 & 1.47 & $\pm 0.05 \pm 0.07$ \\
\hline 0.145 & 1.26 & $\pm 0.05 \pm 0.07$ \\
\hline 0.155 & 1.11 & $\pm 0.05 \pm 0.10$ \\
\hline 0.165 & 0.86 & $\pm 0.04 \pm 0.06$ \\
\hline 0.175 & 0.64 & $\pm 0.03 \pm 0.03$ \\
\hline 0.185 & 0.56 & $\pm 0.03 \pm 0.04$ \\
\hline 0.195 & 0.41 & $\pm 0.03 \pm 0.03$ \\
\hline 0.205 & 0.35 & $\pm 0.03 \pm 0.03$ \\
\hline 0.215 & 0.23 & $\pm 0.02 \pm 0.02$ \\
\hline 0.225 & 0.20 & $\pm 0.02 \pm 0.02$ \\
\hline 0.235 & 0.105 & $\pm 0.014 \pm 0.009$ \\
\hline 0.245 & 0.044 & $\pm 0.008 \pm 0.015$ \\
\hline 0.255 & 0.034 & $\pm 0.009 \pm 0.003$ \\
\hline 0.265 & 0.008 & $\pm 0.003 \pm 0.006$ \\
\hline 0.275 & 0.003 & $\pm 0.002 \pm 0.002$ \\
\hline 0.285 & 0.004 & $\pm 0.004 \pm 0.002$ \\
\hline & & \\
\hline
\end{tabular}

Table 3: Heavy jet mass distribution (mass definition) corrected for detector acceptance and initial state photon radiation. 


\begin{tabular}{|c|c|}
\hline$B$ & $\frac{1}{\sigma_{\text {tot }}} \frac{\Delta \sigma}{\Delta B} \pm($ stat $) \pm(\exp )$ \\
\hline 0.015 & $0.04 \pm 0.01 \pm 0.02$ \\
\hline 0.025 & $0.30 \pm 0.02 \pm 0.09$ \\
\hline 0.035 & $1.95 \pm 0.06 \pm 0.16$ \\
\hline 0.045 & $5.66 \pm 0.09 \pm 0.07$ \\
\hline 0.055 & $9.4 \pm 0.1 \pm 0.3$ \\
\hline 0.065 & $10.6 \pm 0.1 \pm 0.2$ \\
\hline 0.075 & $10.0 \pm 0.1 \pm 0.1$ \\
\hline 0.085 & $8.2 \pm 0.1 \pm 0.2$ \\
\hline 0.095 & $7.1 \pm 0.1 \pm 0.1$ \\
\hline 0.105 & $5.86 \pm 0.10 \pm 0.08$ \\
\hline 0.115 & $5.32 \pm 0.10 \pm 0.13$ \\
\hline 0.125 & $4.49 \pm 0.09 \pm 0.07$ \\
\hline 0.135 & $3.88 \pm 0.08 \pm 0.11$ \\
\hline 0.145 & $3.34 \pm 0.08 \pm 0.08$ \\
\hline 0.155 & $3.07 \pm 0.07 \pm 0.05$ \\
\hline 0.165 & $2.53 \pm 0.07 \pm 0.05$ \\
\hline 0.175 & $2.24 \pm 0.06 \pm 0.07$ \\
\hline 0.185 & $2.01 \pm 0.06 \pm 0.05$ \\
\hline 0.195 & $1.79 \pm 0.06 \pm 0.06$ \\
\hline 0.205 & $1.60 \pm 0.05 \pm 0.04$ \\
\hline 0.215 & $1.41 \pm 0.05 \pm 0.07$ \\
\hline 0.225 & $1.21 \pm 0.05 \pm 0.06$ \\
\hline 0.235 & $1.03 \pm 0.04 \pm 0.03$ \\
\hline 0.245 & $0.90 \pm 0.04 \pm 0.03$ \\
\hline 0.255 & $0.79 \pm 0.04 \pm 0.03$ \\
\hline 0.265 & $0.65 \pm 0.03 \pm 0.01$ \\
\hline 0.275 & $0.61 \pm 0.03 \pm 0.06$ \\
\hline 0.285 & $0.50 \pm 0.03 \pm 0.03$ \\
\hline 0.295 & $0.40 \pm 0.03 \pm 0.02$ \\
\hline
\end{tabular}

Table 4: Total jet broadening distribution corrected for detector acceptance and initial state photon radiation. 


\begin{tabular}{|c||cc|}
\hline$B_{W}$ & $\frac{1}{\sigma_{\text {tot }}} \frac{\Delta \sigma}{\Delta B_{W}} \pm(\mathrm{stat}) \pm(\mathrm{exp})$ \\
\hline 0.005 & 0.017 & $\pm 0.007 \pm 0.013$ \\
\hline 0.015 & 0.88 & $\pm 0.04 \pm 0.12$ \\
\hline 0.025 & 8.9 & $\pm 0.1 \pm 0.3$ \\
\hline 0.035 & 15.9 & $\pm 0.2 \pm 0.3$ \\
\hline 0.045 & 13.7 & $\pm 0.2 \pm 0.3$ \\
\hline 0.055 & 10.3 & $\pm 0.1 \pm 0.2$ \\
\hline 0.065 & 8.3 & $\pm 0.1 \pm 0.1$ \\
\hline 0.075 & 6.65 & $\pm 0.11 \pm 0.08$ \\
\hline 0.085 & 5.51 & $\pm 0.10 \pm 0.07$ \\
\hline 0.095 & 4.65 & $\pm 0.09 \pm 0.09$ \\
\hline 0.105 & 3.91 & $\pm 0.08 \pm 0.07$ \\
\hline 0.115 & 3.36 & $\pm 0.08 \pm 0.05$ \\
\hline 0.125 & 2.80 & $\pm 0.07 \pm 0.06$ \\
\hline 0.135 & 2.34 & $\pm 0.06 \pm 0.03$ \\
\hline 0.145 & 2.02 & $\pm 0.06 \pm 0.08$ \\
\hline 0.155 & 1.77 & $\pm 0.06 \pm 0.06$ \\
\hline 0.165 & 1.47 & $\pm 0.05 \pm 0.04$ \\
\hline 0.175 & 1.28 & $\pm 0.05 \pm 0.03$ \\
\hline 0.185 & 1.10 & $\pm 0.04 \pm 0.04$ \\
\hline 0.195 & 0.87 & $\pm 0.04 \pm 0.07$ \\
\hline 0.205 & 0.69 & $\pm 0.03 \pm 0.03$ \\
\hline 0.215 & 0.61 & $\pm 0.03 \pm 0.04$ \\
\hline 0.225 & 0.49 & $\pm 0.03 \pm 0.02$ \\
\hline 0.235 & 0.34 & $\pm 0.02 \pm 0.02$ \\
\hline 0.245 & 0.25 & $\pm 0.02 \pm 0.03$ \\
\hline 0.255 & 0.16 & $\pm 0.02 \pm 0.01$ \\
\hline 0.265 & 0.11 & $\pm 0.01 \pm 0.01$ \\
\hline 0.275 & 0.043 & $\pm 0.007 \pm 0.011$ \\
\hline 0.285 & 0.018 & $\pm 0.005 \pm 0.005$ \\
\hline 0.295 & 0.009 & $\pm 0.004 \pm 0.002$ \\
\hline & & \\
\hline
\end{tabular}

Table 5: Wide jet broadening distribution corrected for detector acceptance and initial state photon radiation. 


\begin{tabular}{|c||cc|}
\hline$\chi_{E E C}$ & $E E C\left(\chi_{E E C}\right) \pm($ stat $) \pm(\exp )$ \\
\hline 91.8 & 0.0785 & $\pm 0.0007 \pm 0.0020$ \\
\hline 95.4 & 0.0800 & $\pm 0.0007 \pm 0.0015$ \\
\hline 99.0 & 0.0814 & $\pm 0.0008 \pm 0.0015$ \\
\hline 102.6 & 0.0839 & $\pm 0.0008 \pm 0.0010$ \\
\hline 106.2 & 0.0863 & $\pm 0.0008 \pm 0.0018$ \\
\hline 109.8 & 0.0899 & $\pm 0.0008 \pm 0.0009$ \\
\hline 113.4 & 0.0956 & $\pm 0.0009 \pm 0.0012$ \\
\hline 117.0 & 0.1001 & $\pm 0.0009 \pm 0.0017$ \\
\hline 120.6 & 0.1061 & $\pm 0.0009 \pm 0.0016$ \\
\hline 124.2 & 0.115 & $\pm 0.001 \pm 0.002$ \\
\hline 127.8 & 0.124 & $\pm 0.001 \pm 0.001$ \\
\hline 131.4 & 0.137 & $\pm 0.001 \pm 0.002$ \\
\hline 135.0 & 0.152 & $\pm 0.001 \pm 0.001$ \\
\hline 138.6 & 0.169 & $\pm 0.001 \pm 0.002$ \\
\hline 142.2 & 0.194 & $\pm 0.001 \pm 0.003$ \\
\hline 145.8 & 0.225 & $\pm 0.001 \pm 0.003$ \\
\hline 149.4 & 0.264 & $\pm 0.002 \pm 0.004$ \\
\hline 153.0 & 0.317 & $\pm 0.002 \pm 0.004$ \\
\hline 156.6 & 0.390 & $\pm 0.002 \pm 0.004$ \\
\hline 160.2 & 0.492 & $\pm 0.003 \pm 0.004$ \\
\hline 163.8 & 0.632 & $\pm 0.003 \pm 0.008$ \\
\hline 167.4 & 0.832 & $\pm 0.004 \pm 0.012$ \\
\hline 171.0 & 1.086 & $\pm 0.005 \pm 0.017$ \\
\hline 174.6 & 1.269 & $\pm 0.007 \pm 0.012$ \\
\hline 178.2 & 0.750 & $\pm 0.006 \pm 0.051$ \\
\hline & & \\
\hline
\end{tabular}

Table 6: Distribution of the back-to-back energy-energy correlation corrected for detector acceptance and initial state photon radiation. 


\begin{tabular}{|c|c|}
\hline Variable & $y_{\max }$ \\
\hline $1-T$ & $\frac{1}{2}$ \\
\hline$\rho^{(T)}$ & $\frac{1}{2}$ \\
\hline$\rho^{(M)}$ & $\frac{1}{3}$ \\
\hline$B$ & 0.5 \\
\hline$B_{W}$ & 0.33 \\
\hline$(1+\cos \chi) / 2$ & $\frac{1}{2}$ \\
\hline
\end{tabular}

Table 7: Upper kinematic boundary limits. For the jet broadening variables the limits were estimated numerically with a Monte Carlo simulation.

\begin{tabular}{|l||c|c|c|}
\hline Variable & Bin $\Delta y$ & $\frac{1}{\sigma_{\text {tot }}} \frac{\Delta \sigma}{\Delta y}$ & $\alpha_{S}$ \\
\hline $1-T$ & $0.02 \ldots 0.03$ & $16.2 \pm 0.8$ & $0.122 \pm 0.008$ \\
\hline$\rho^{(T)}$ & $0.02 \ldots .0 .03$ & $20.2 \pm 0.5$ & $0.117 \pm 0.008$ \\
\hline$\rho^{(M)}$ & $0.02 \ldots 0.03$ & $20.6 \pm 0.7$ & $0.119 \pm 0.007$ \\
\hline$B$ & $0.05 \ldots 0.06$ & $9.4 \pm 0.3$ & $0.116 \pm 0.007$ \\
\hline$B_{W}$ & $0.04 \ldots 0.05$ & $13.7 \pm 0.4$ & $0.113 \pm 0.010$ \\
\hline
\end{tabular}

Table 8: Bin edges and contents used for method 1 and results for $\alpha_{S}$ at the scale $\mu^{2}=M_{\mathrm{Z}}^{2}$. The quoted errors of the data points include statistical and experimental uncertainties, and the errors of the $\alpha_{S}$ values include theoretical uncertainties additionally.

\begin{tabular}{|l||c|c|c||c|c|}
\hline Variable & Fit Range & $\alpha_{S}(\ln R)$ & $\alpha_{S}\left(R-G_{21}\right)$ & Fit Range & $\alpha_{S}(R)$ \\
\hline $1-T$ & $0.06 \ldots 0.30$ & $0.122 \pm 0.006$ & $0.123 \pm 0.007$ & $0.10 \ldots 0.30$ & $0.128 \pm 0.006$ \\
\hline$\rho^{(T)}$ & $0.03 \ldots 0.24$ & $0.123 \pm 0.009$ & $0.124 \pm 0.009$ & $0.08 \ldots 0.24$ & $0.125 \pm 0.009$ \\
\hline$\rho^{(M)}$ & $0.02 \ldots 0.15$ & $0.124 \pm 0.007$ & $0.126 \pm 0.009$ & $0.08 \ldots 0.15$ & $0.128 \pm 0.011$ \\
\hline$B$ & $0.06 \ldots 0.24$ & $0.121 \pm 0.009$ & $0.117 \pm 0.009$ & $0.10 \ldots 0.24$ & $0.133 \pm 0.013$ \\
\hline$B_{W}$ & $0.04 \ldots 0.20$ & $0.110 \pm 0.008$ & $0.107 \pm 0.008$ & $0.08 \ldots 0.20$ & $0.122 \pm 0.008$ \\
\hline$E E C$ & $104.4^{\circ} \ldots 162.0^{\circ}$ & $0.134 \pm 0.007$ & $0.135 \pm 0.007$ & $104.4^{\circ} \ldots 136.8^{\circ}$ & $0.126 \pm 0.007$ \\
\hline
\end{tabular}

Table 9: Results of method 2 for the scale $\mu^{2}=M_{\mathrm{Z}}^{2}$. The quoted errors are the total errors including theoretical uncertainties as found in an analysis using each event shape variable separately. For the $R$-scheme, which is used in this analysis only to estimate the systematic uncertainty, a restricted fit range is used. 


\section{Figure Captions}

\section{Figure 1:}

a) Ratio of the resummed leading and next-to-leading logarithms to $H_{1} \alpha_{S}+H_{2} \alpha_{S}^{2}$ of equations (18) and (19) versus $1-T$. For low values of $1-T$, where this ratio becomes large, the theory is dominated by resummed logarithms.

b) Ratio of the next-to-leading logarithms to the leading logarithms. If this ratio is small NLLA should be reliable.

c) Ratio of the only known subleading logarithm $G_{21} \ln y$ and $G_{22} \ln ^{2} y$. If this ratio is small one expects a small matching scheme ambiguity.

Figure 2: As Figure 2 but for the back-to-back energy-energy correlation EEC.

\section{Figure 3:}

Upper plot: Measured thrust distribution corrected for acceptance and initial state radiation. The histogram presents the result of a fit to the data of the combined theory with $\ln R$-matching scheme at $\mu^{2}=M_{\mathrm{Z}}^{2}$ as described in the text.

Middle plot: Bin-by-bin detector correction including effects due to initial state radiation.

Lower plot: Size of the hadronization correction. The width of the band indicates the uncertainty of the correction.

Figure 4: As Figure 3 but for the heavy jet mass (thrust definition).

Figure 5: As Figure 3 but for the heavy jet mass (mass definition).

Figure 6: As Figure 3 but for the total jet broadening variable.

Figure 7: As Figure 3 but for the wide jet broadening variable.

Figure 8: As Figure 3 but for the back-to-back energy-energy correlation EEC.

\section{Figure 9:}

Upper plot: Values of $\alpha_{S}\left(M_{\mathrm{Z}}^{2}\right)$ obtained at different scales $\mu^{2} / M_{\mathrm{Z}}^{2}$ from fits to the distributions in thrust, heavy jet masses, jet broadening variables and back-to-back energyenergy correlation. All fits are performed by using the $\ln R$-scheme.

Lower plot: Combined $\alpha_{S}$ values as a function of the scale $\mu^{2} / M_{\mathrm{Z}}^{2}$ using the $\ln R$-scheme. The width of the band corresponds to the full experimental and theoretical error of the combined value. 

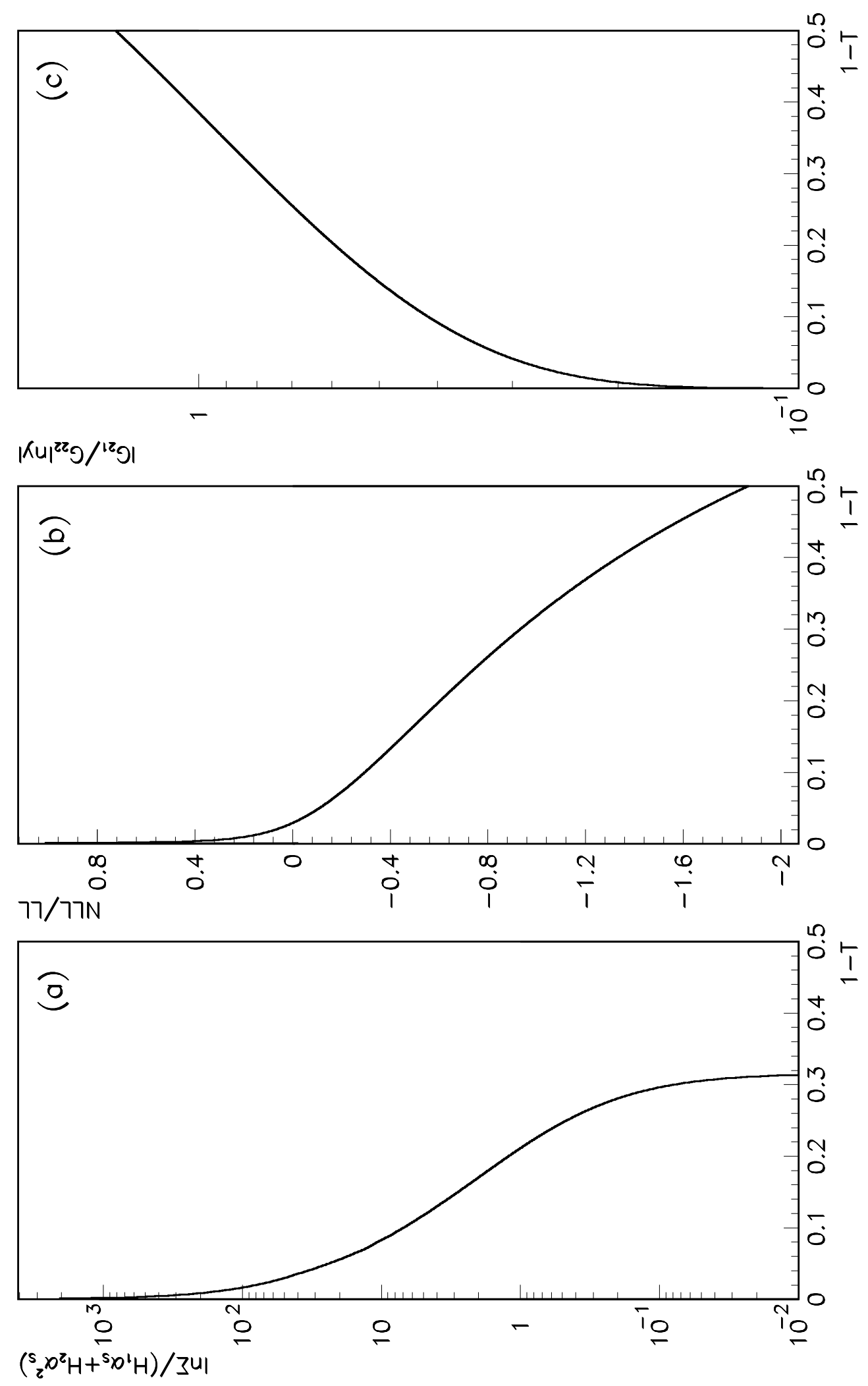

Figure 1: 

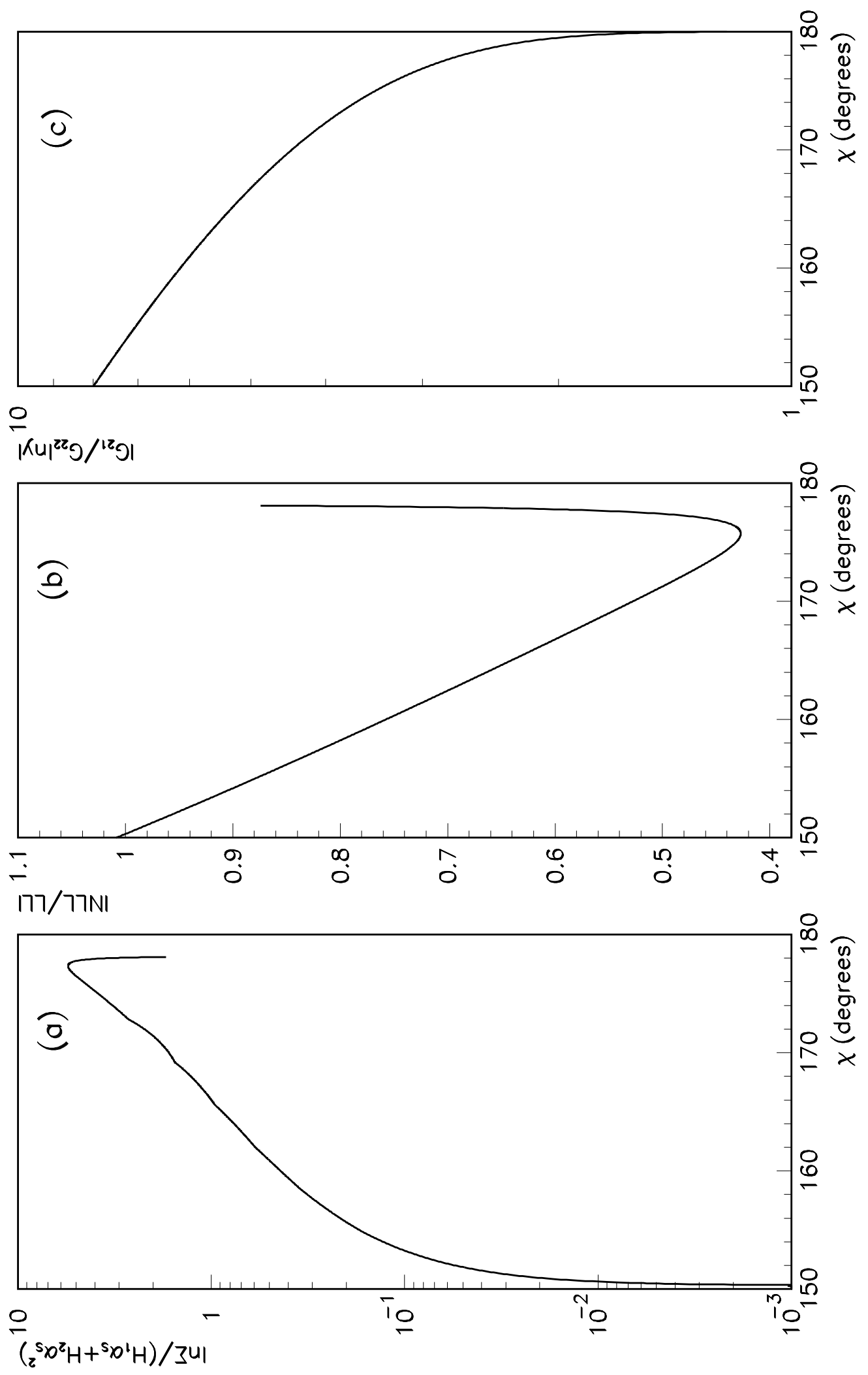

Figure 2: 


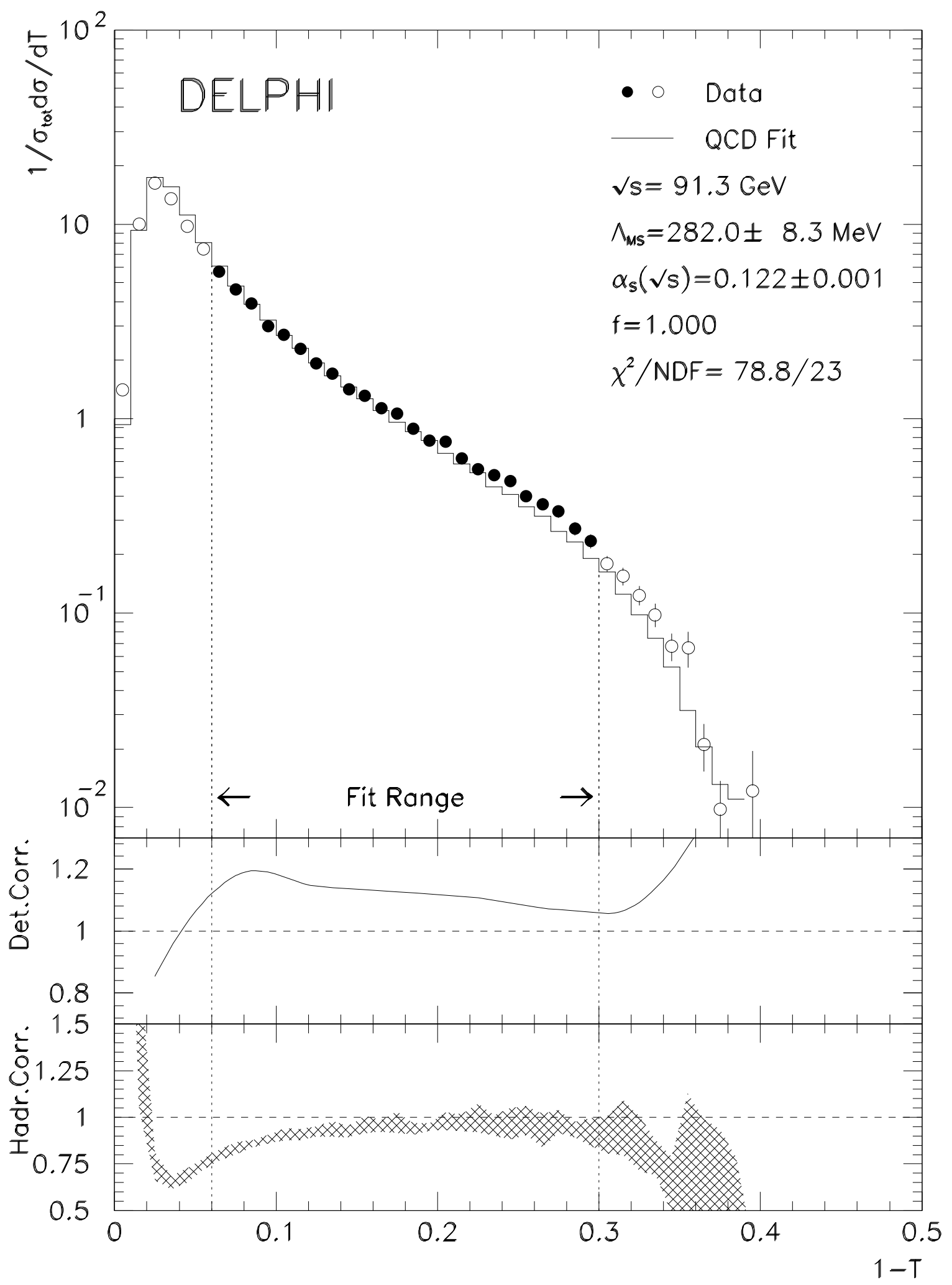

Figure 3: 


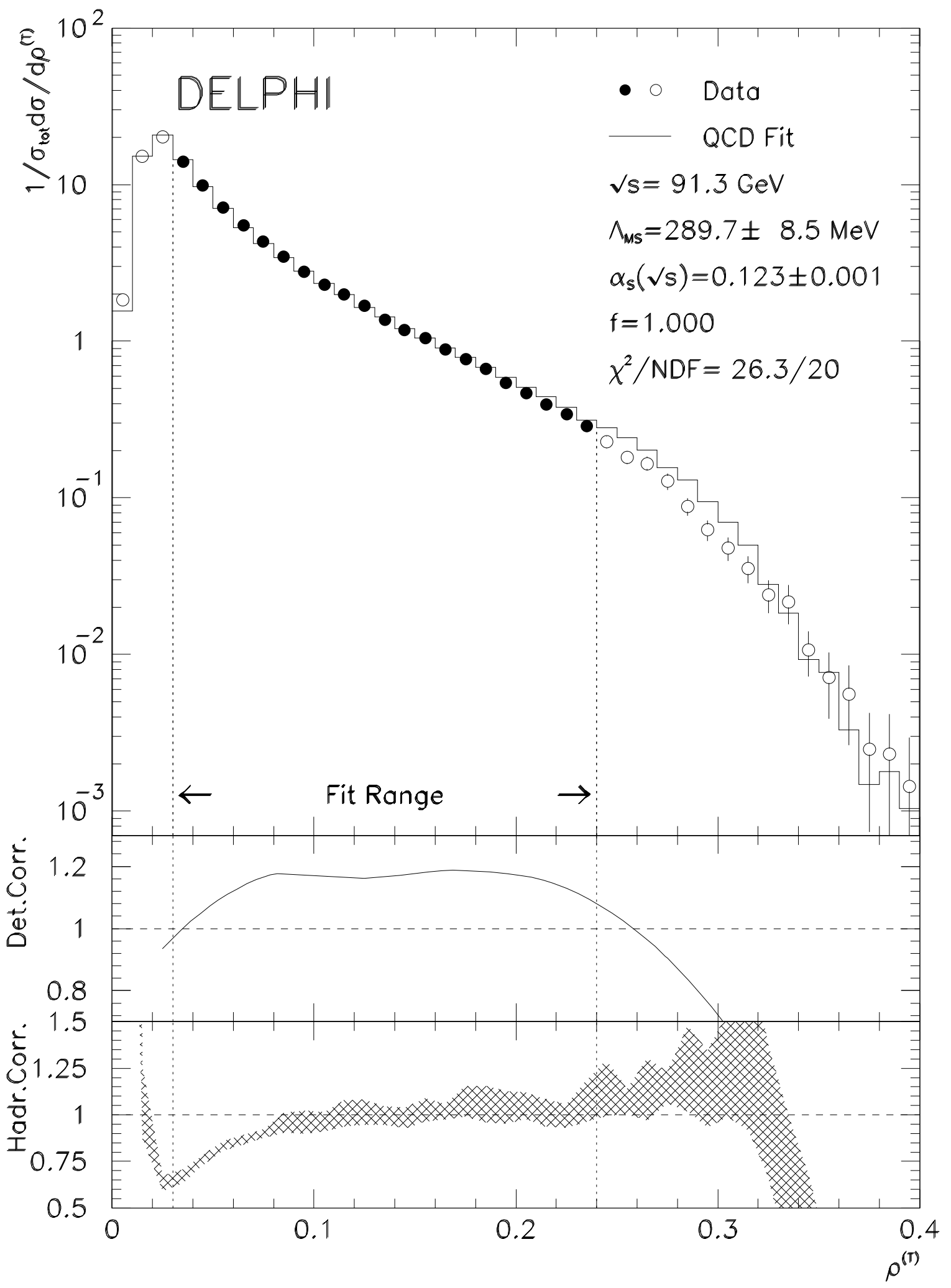

Figure 4: 


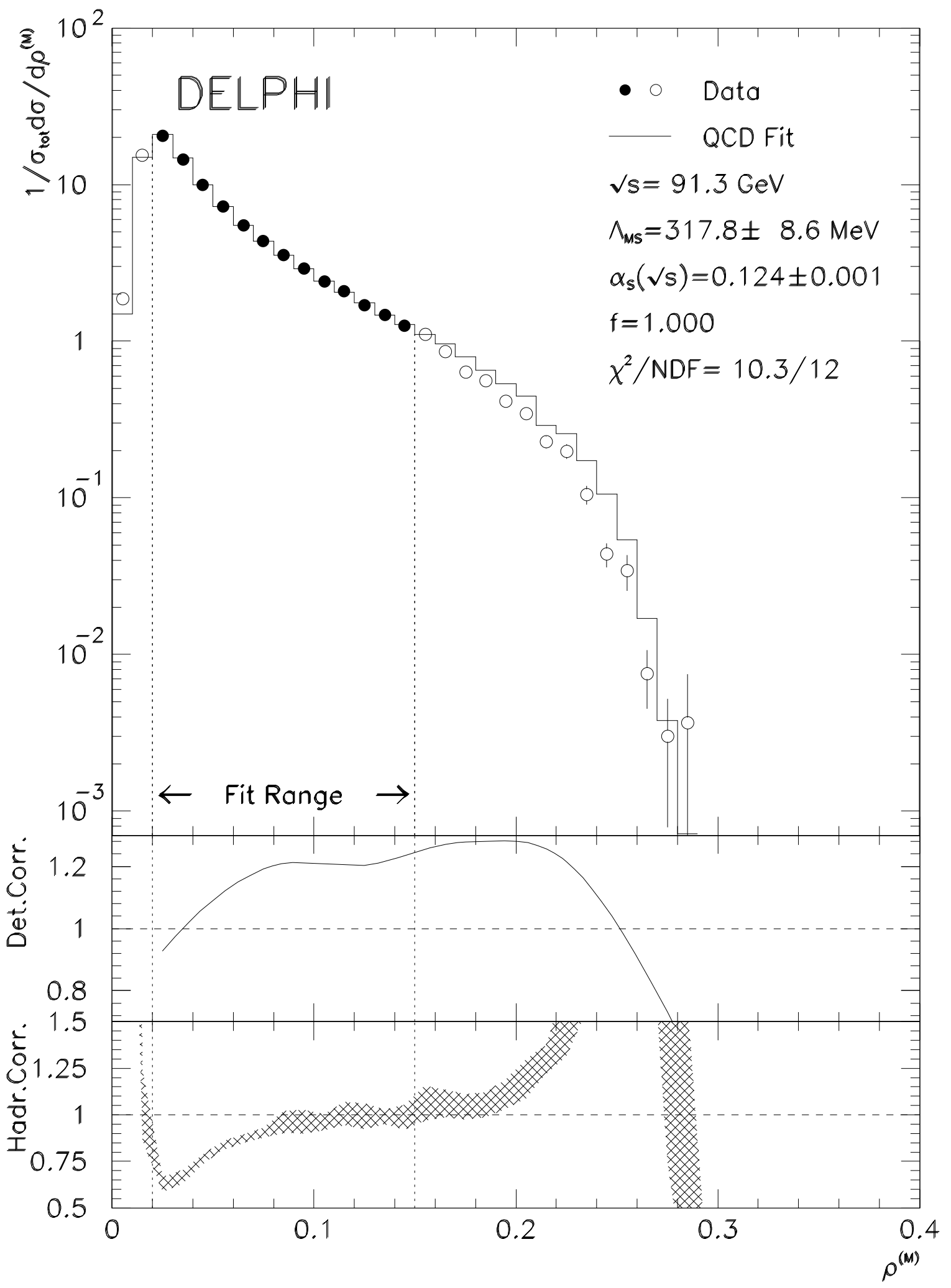

Figure 5: 


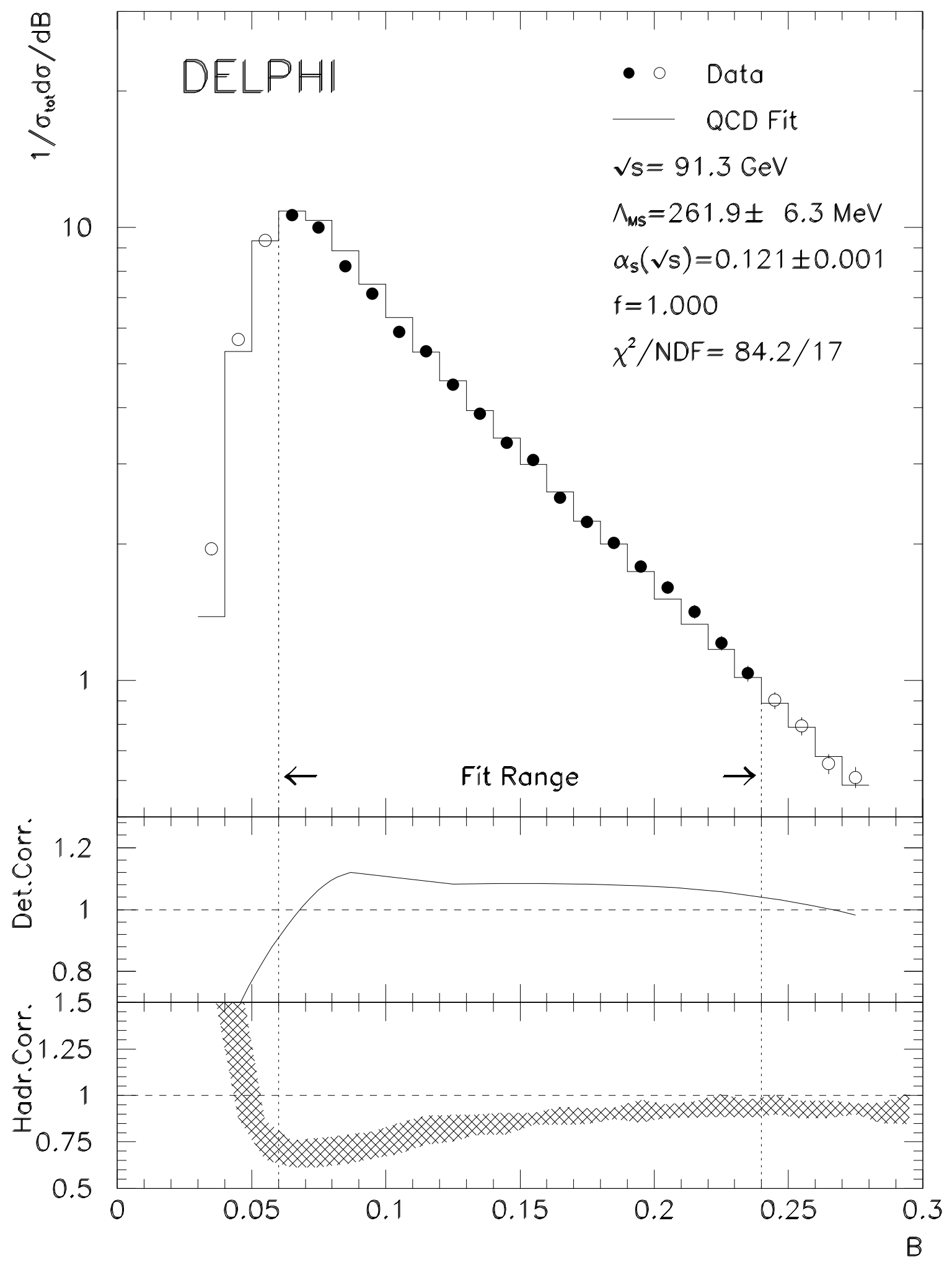

Figure 6: 


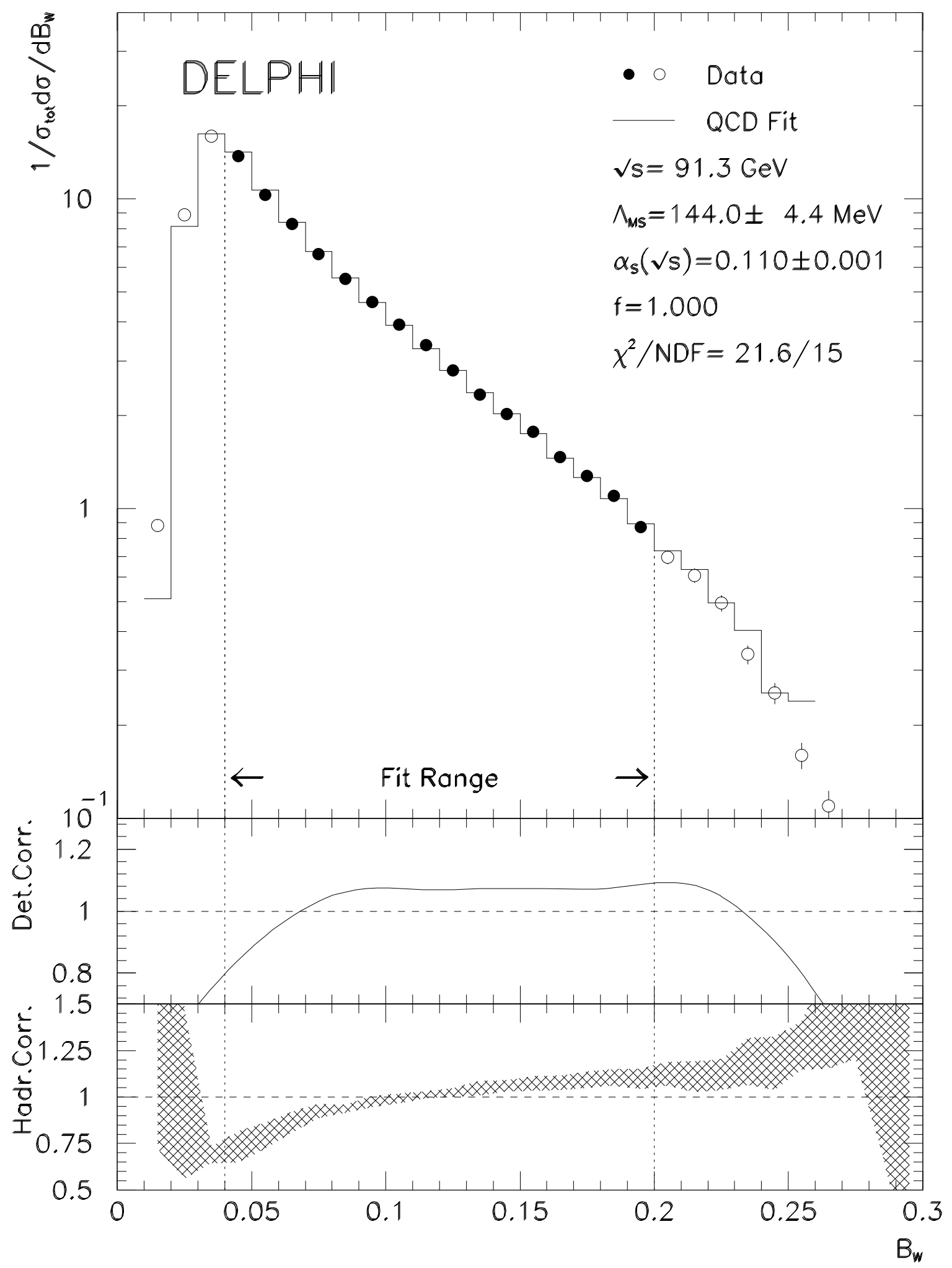

Figure 7: 


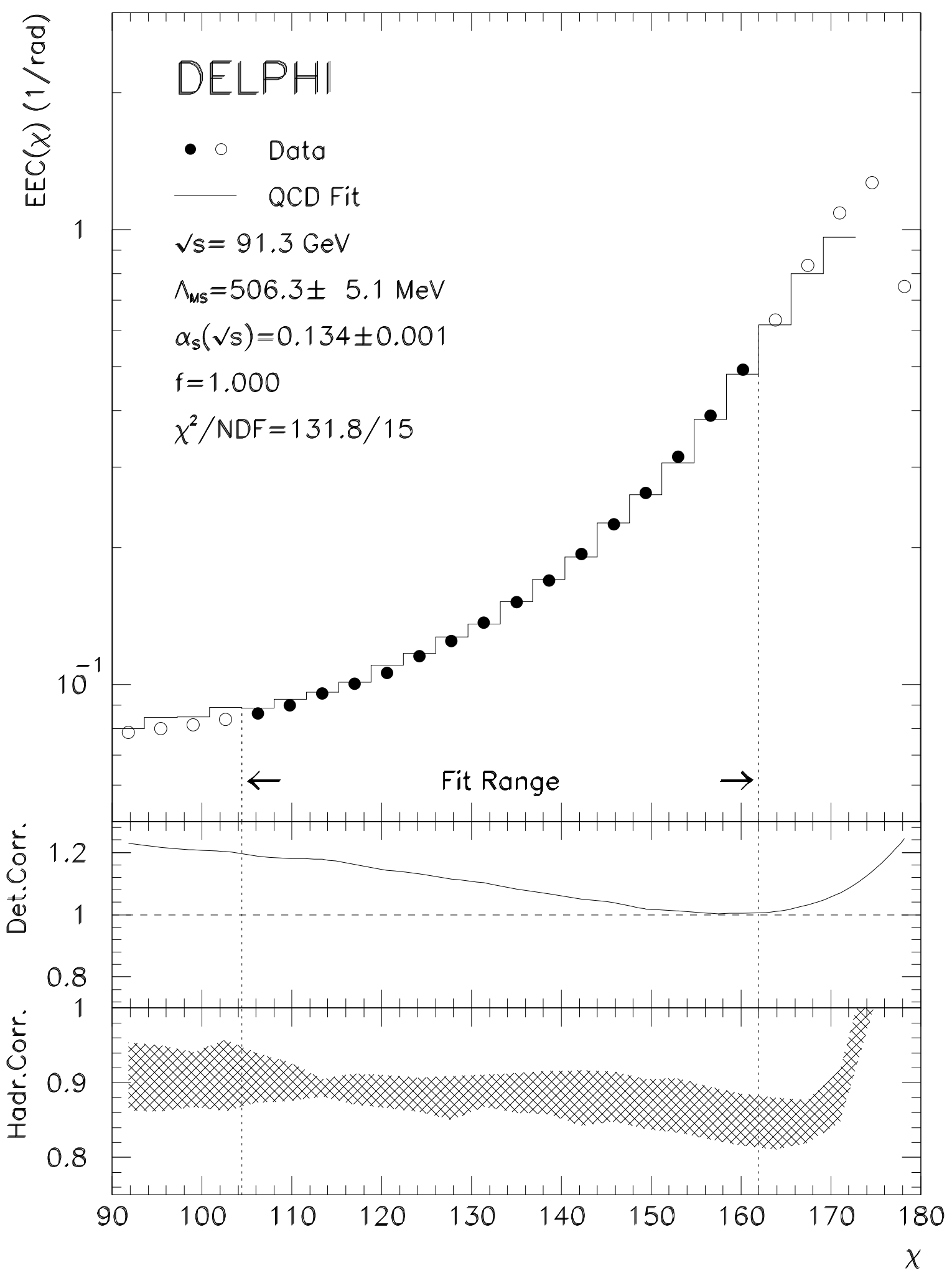

Figure 8: 


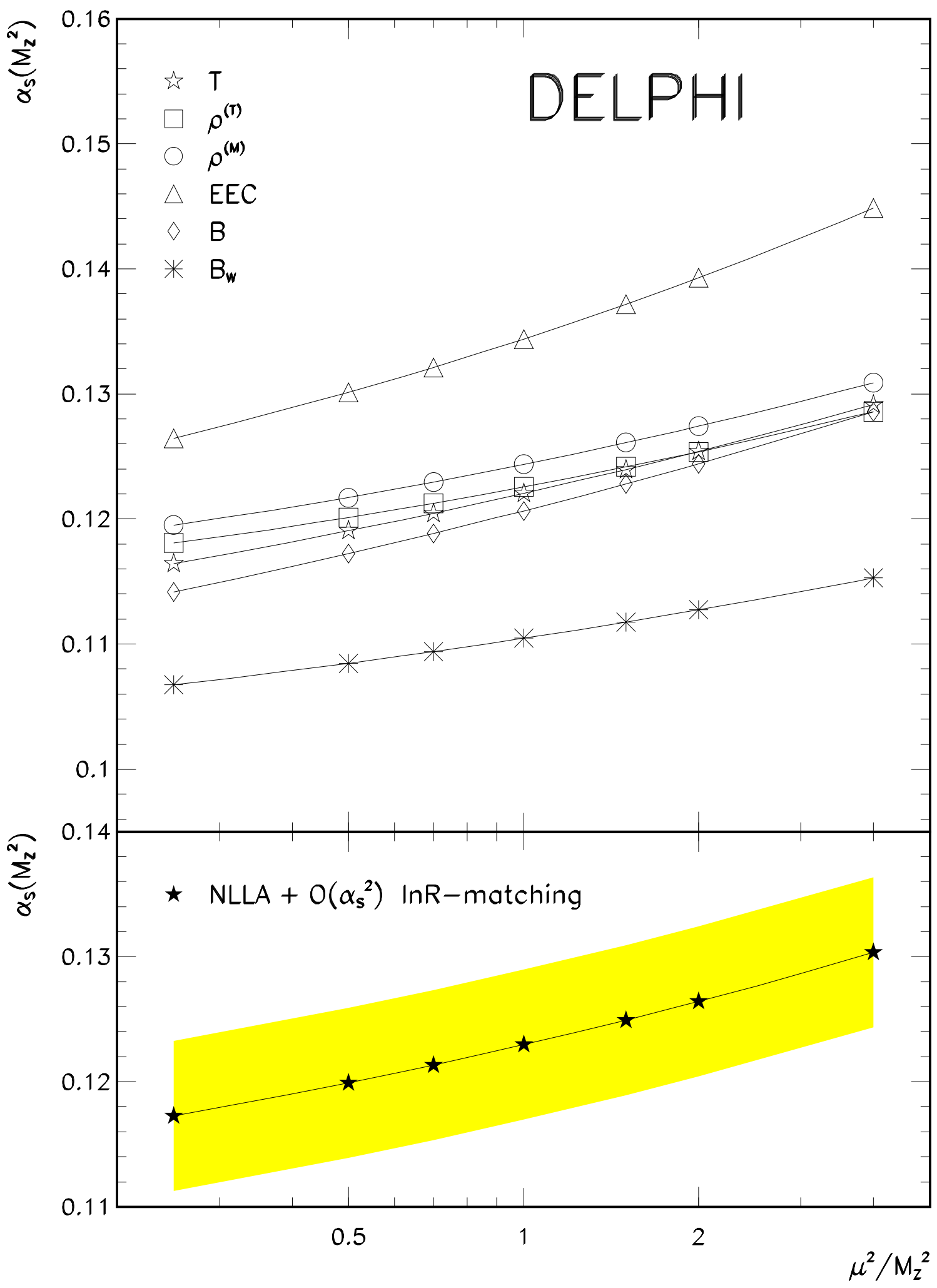

Figure 9: 